\title{
Big Brother beim Lernen: Privatsphäre und Datenschutz in Lernplattformen
}

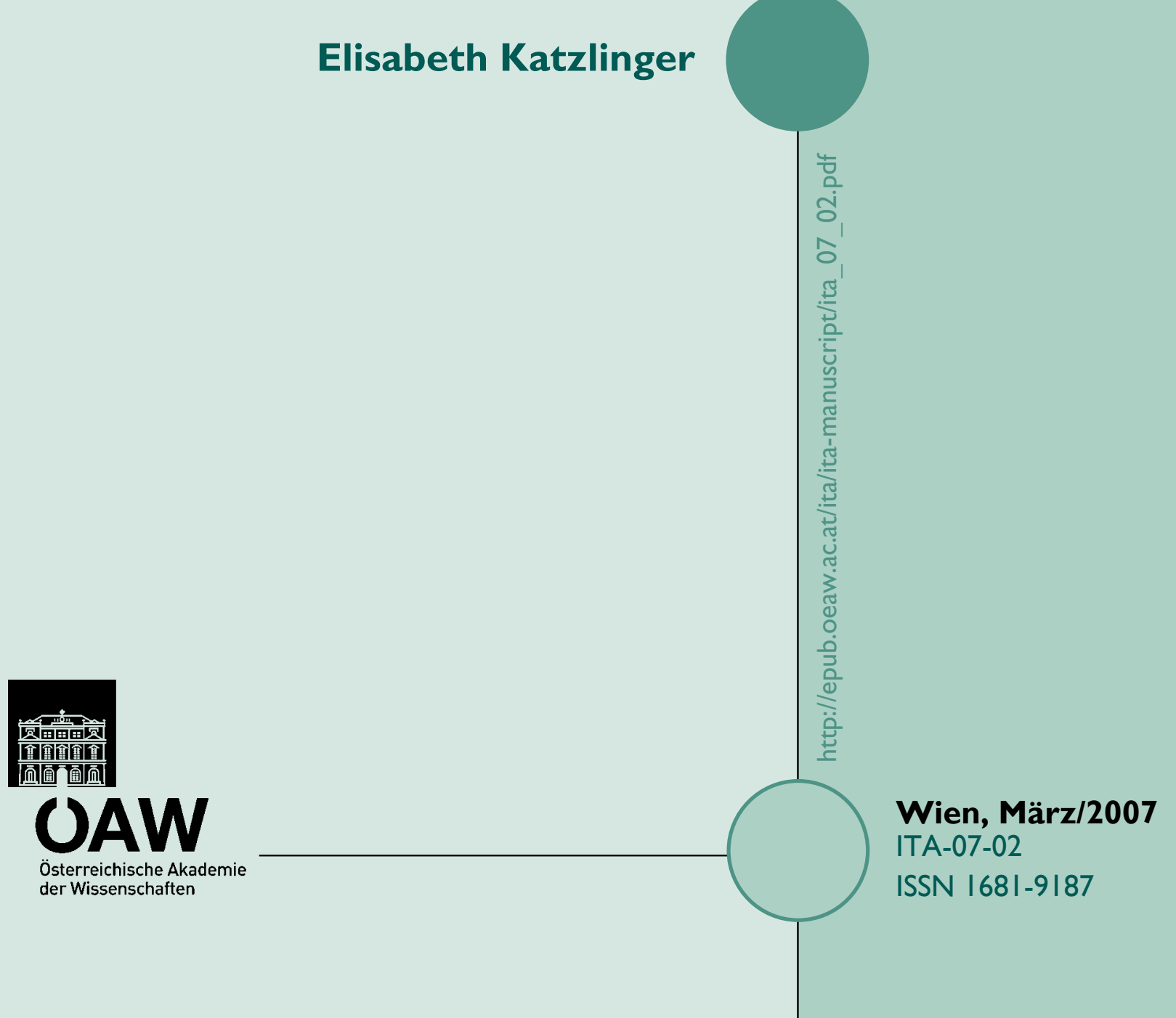





\title{
Big Brother beim Lernen: Privatsphäre und Datenschutz in Lernplattformen
}

\section{Elisabeth Katzlinger}

Institut für Datenverarbeitung in den Sozial- und Wirtschaftswissenschaften, Johannes Kepler Universität Linz.

\section{Keywords}

Lernplattformen; E-Learning; Privatsphäre; Datenschutz; User profiling; User tracking

\begin{abstract}
Für die Unterstützung von Lernprozessen werden Lernplattformen wie Moodle oder Blackboard, aber auch Social Software wie Weblogs, Wikis, Diskussionsforen oder Chats verstärkt eingesetzt. In diesen Anwendungen werden die Zugangsdaten und die Daten über die einzelnen Aktivitäten aller Beteiligten gespeichert und stehen für Auswertungen zur Verfügung. Mit unterschiedlichen Methoden der Datenauswertung, wie User Profiling, User Tracking oder Text Mining können diese Daten ausgewertet werden und beispielsweise für die Beurteilung der Lernenden oder die Überprüfung der Beteiligung an einer Lehrveranstaltung heran gezogen werden. Dabei kann die Privatsphäre und der Schutz der personenbezogenen Daten verletzt werden. Die vorliegende Arbeit beschäftigt sich mit den Auswertungsmöglichkeiten von Benutzerdaten in Lernplattformen und den sich daraus ergebenden Problembereichen.
\end{abstract}




\section{Inhalt}

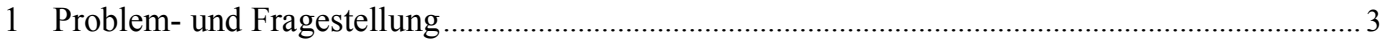

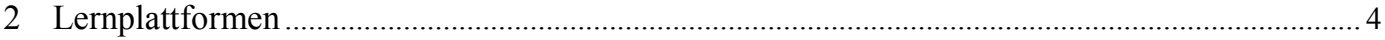

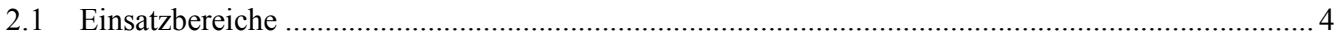

2.2 Komponenten von Lernplattformen ........................................................................... 4

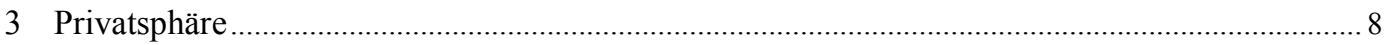

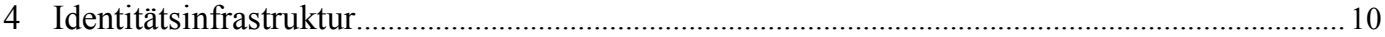

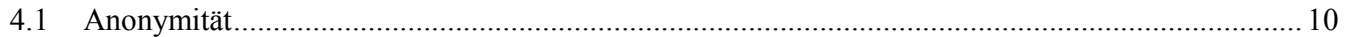

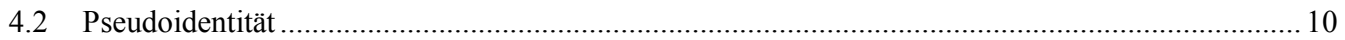

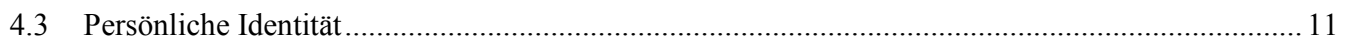

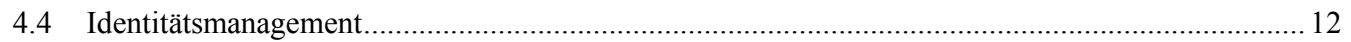

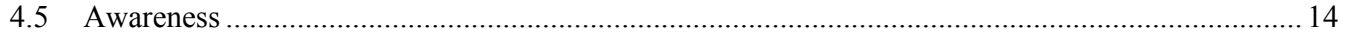

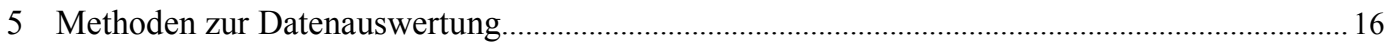

5.1 User Profiling .......................................................................................................................... 16

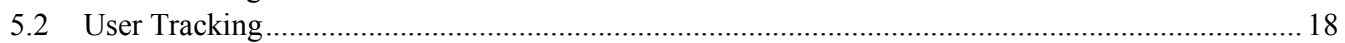

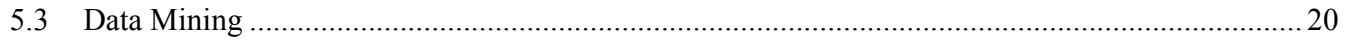

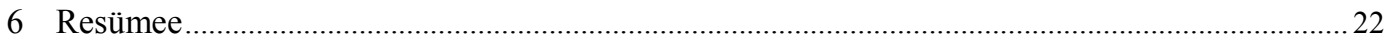

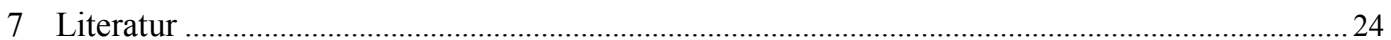

Dieser Beitrag basiert auf dem Vortrag „Big Brother beim Lernen: Datensammlung in Lernplattformen “ im Rahmen der Sechsten Österreichischen TA-Konferenz „TA'06: Vermessen, codiert, entschlüsselt? Potenziale und Risken der zunehmenden Datenverfügbarkeit“, Wien, 29. Mai 2006.

\section{IMPRESSUM}

\section{Medieninhaber:}

Österreichische Akademie der Wissenschaften

Juristische Person öffentlichen Rechts (BGBI 569/|92I idF BGB| I |30/2003)

Dr. Ignaz Seipel-Platz 2, A-10 10 Wien

\section{Herausgeber:}

Institut für Technikfolgen-Abschätzung (ITA)

Strohgasse 45/5, A- 1030 Wien

http://www.oeaw.ac.at/ita

Die ITA-manu:scripts erscheinen unregelmäßig und dienen der Veröffentlichung

von Arbeitspapieren und Vorträgen von Institutsangehörigen und Gästen.

Die manu:scripts werden ausschließlich über das Internetportal „epub.oeaw“

der Öffentlichkeit zur Verfügung gestellt:

http://epub.oeaw.ac.at/ita/ita-manuscript

ITA-manuscript Nr.: ITA-07-02 (März/2007)

ISSN-online: | 8| 8-6556

http://epub.oeaw.ac.at/ita/ita-manuscript/ita_07_02.pdf

c 2007 ITA - Alle Rechte vorbehalten 


\section{Problem- und Fragestellung}

Lernplattformen wie Blackboard oder Moodle, aber auch Social Software wie Weblogs, Wikis, Diskussionsforen oder Chats werden zur Unterstützung von Lernprozessen verstärkt eingesetzt. Um einen reibungslosen Ablauf des Betriebes der Informations- und Kommunikationssysteme zu ermöglichen, werden die Zugangsdaten und Daten über die einzelnen Aktivitäten aller Beteiligten, sowohl der Lernenden als auch der Lehrenden, aufgezeichnet. Diese Daten dienen vordergründig zur technischen Überwachung der Systeme. Die Menge der gesammelten Daten und deren Verwendungsmöglichkeiten beinhalten neue, versteckte und offene Risiken für die Privatsphäre der Beteiligten.

Die Methoden des User Trackings und User Profilings sind aus den Bereichen des E-Business wie beispielsweise bei CRM-Systemen (Kundenbeziehungsmanagement) hinlänglich bekannt. Aus der Analyse des Click- und Downloadverhaltens wird ein individuelles Profil erstellt, das für Marketingaktivitäten genutzt werden kann (Pils 2004, 336). Bei der Anwendung dieser Methoden auf Lernende ergibt sich eine Reihe von zusätzlichen Problemen.

Die vorliegende Arbeit beschäftigt sich mit Auswertungsmöglichkeiten von Benutzerdaten in Lernplattformen und in Social Software, die in der Lehre eingesetzt werden, sowie deren Problembereichen. Die Erstellung von Benutzerprofilen und auch Gruppenprofilen wird durch die Lernplattformen unterstützt. Wofür diese Benutzerprofile verwendet werden, liegt in der Hand der Lehrenden, sie können beispielsweise für die Verbesserung des Lernarrangements, Analyse der Kommunikationsbeziehungen oder als Beurteilungsgrundlage herangezogen werden.

Im Rahmen einer Lehrveranstaltung Informationsverarbeitung kam die Lernplattform Moodle zum Einsatz. In einer begleitenden Studie wurde der Frage nachgegangen, wie weit die quantitative Auswertung der Zugangsdaten für die Beurteilung des Lehrveranstaltungserfolges dienen kann. Neben der Organisation der Lehrveranstaltung wurde die Verteilung von Lernunterlagen und die Abgabe von den Arbeiten der Studierenden über Moodle abgewickelt. Die Lernplattform stand den Studierenden auch zur Organisation ihrer Gruppenarbeiten zur Verfügung. Weiters wurden die Instrumente „Lernchat“ und „Forum“ eingesetzt. Durch den Einsatz dieses Lernszenarios konnten personenbezogene Daten gesammelt werden, die sich mit quantitativen Methoden auswerten lassen.

Im Zuge der Evaluierung der Lehrveranstaltung wurde Augenmerk auf die Einstellung der Studierenden zu dieser Datensammlung gelegt. Die erste Auswertung der Stellungnahmen der Studierenden zeigte eine sehr ambivalente Einstellung zur statistischen Analyse ihrer Benutzerdaten. Auf der einen Seite wird sie als objektives Maß für die Beurteilung des Lehrveranstaltungserfolges begrüßt, auf der anderen wird die Verhaltenskontrolle negativ beurteilt. Ein weiteres Problem ergibt sich aus der Durchschaubarkeit des Systems: einem Teil der Studierenden war lange Zeit nicht bewusst, dass alle ihre Aktivitäten im System aufgezeichnet werden und jederzeit ausgewertet werden können. Erst im Zuge der Thematisierung innerhalb der Lehrveranstaltung wurde ihnen klar, dass mit der Aufzeichnung der Daten ihr Verhalten kontrolliert und so für die Beurteilung ihres Lernerfolges herangezogen werden kann. Dies bewirkte eine Verhaltensänderung der Studierenden in der Weise, dass sich die Aktivitäten im System schlagartig erhöhten.

Von Seiten der Lehrenden wurde es begrüßt, dass „objektive Daten“ über die Beteiligung der einzelnen Studierenden und der Lerngruppen an der Lehrveranstaltung zur Verfügung standen. Es war naheliegend, dass diese Daten auch für die Beurteilung herangezogen wurden, ohne genauer der Frage nach zu gehen, ob sie den Lernerfolg widerspiegeln. 


\section{Lernplattformen}

Den technischen Kern einer E-Learning Infrastruktur bilden die Lernplattformen, Lernumgebungen oder Learning Management Systeme ${ }^{1}$. Sie stellen die Basisdienste für die Lehrenden und die Lernenden zur Verfügung und haben neben der Unterstützungsfunktion meist auch eine Anregungsfunktion. „Unter einer webbasierten Lernplattform ist eine serverseitig installierte Software zu verstehen, die beliebige Lerninhalte über das Internet zu vermitteln hilft und die Organisation der dabei notwendigen Lernprozesse unterstützt“ (Baumgartner et al. 2002, 24).

Im englischsprachigen Raum werden analog für Lernumgebungen die Bezeichnungen LMS (Learning Management System) und LCMS (Learning Content Management System) als auch der Begriff des CMS (Content Management System) verwendet (Baumgartner et al. 2002).

\section{I Einsatzbereiche}

Mit dem Einsatz von Lernplattformen geht eine verstärkte Individualisierung des Lernprozesses einher. Im Vergleich zu herkömmlichen Lernarrangements kommt es zu einer stärkeren Flexibilität von Lernorten und Lernzeiten. Die Lernenden müssen nicht an einem bestimmten Ort sein, der Zugang zu den Lernmaterialien ist mit Computer und Netz von (fast) allen Orten aus möglich. Auch hinsichtlich der Lernzeiten und Lerndauer können die Lernenden frei wählen.

Diese Flexibilität und Variabilität von Lernzeiten und Lernorten öffnet Lernangebote für Personen, die ansonsten von Lernangeboten ausgeschlossen wären. Die gilt insbesondere für den tertiären Bildungsbereich. Gleichzeitig wird das Lernen (auch für berufliche Zwecke) zunehmend in die Privatsphäre verlagert, was zu Vereinsamung und Vereinzelung führen kann (Fischer et al. 2003, 10). Die Integration des Lernens in die häusliche Umgebung hat nicht nur Vorteile, sondern bereitet auch eine Reihe von Schwierigkeiten, wie beispielsweise, dass es keine ungestörte Lernumgebung gibt.

\subsection{Komponenten von Lernplattformen}

Lernplattformen bieten verschiedene Komponenten in integrierter Form an (vgl. Appelt 2004, 137f). Die jeweilig am Markt befindlichen Produkte haben die einzelnen Komponenten unterschiedlich stark ausgeprägt.

\subsection{Kommunikations- und Kooperationswerkzeuge}

Werkzeuge, welche die Kommunikation und Kooperation zwischen den Studierenden und zwischen Studierenden und Lehrenden ermöglichen, bilden eine der zentralen Komponenten einer Lernplattform. Die Kommunikationswerkzeuge ermöglichen eine räumliche Verteilung der Kommunikationspartner, sie können aber auch verwendet werden, wenn die Partner sich in einem Raum aufhalten.

1 Die Begriffe werden zum Teil synonym verwendet. 
Die Kommunikationswerkzeuge lassen sich in zwei Klassen unterteilen:

- Werkzeuge für die synchrone Kommunikation wie: Chat, Videokonferenz, Shared Whiteboard und Instant-Messaging System.

- Werkzenge für die asynchrone Kommunikation wie: Diskussionsforen, Dateiaustausch, E-Mail, Verwaltung von Gruppen, Einrichtung privater und gemeinsamer Arbeitsbereiche, Benachrichtigungssysteme.

Für das kooperative Lernen ist Kommunikation die Grundlage. Das Vorhandensein von Kommunikationswerkzeugen ist die Voraussetzung für das gemeinsame Erarbeiten von Lerninhalten im virtuellen Raum.

\subsubsection{Administrationswerkzeuge}

Für die Verwaltung von Benutzern und Ressourcen steht eine Reihe von Werkzeugen zur Verfügung. Bei der Benutzerregistrierung werden zum Teil sehr umfangreiche Daten über die Beteiligten erfasst. Die Registrierung erfolgt entweder durch Selbstregistrierung durch die Benutzer, durch den Administrator oder durch die Verwendung schon vorhandener Daten aus anderen Informationssystemen. Die Administrationstools unterstützen die Vergabe der Rechte und der Rollen (Administrator, Lehrende, Tutoren, Studierende). Die Vergabe von differenzierten Rechten erfolgt normalerweise rollenbasiert. Die Administrationswerkzeuge unterstützen unterschiedliche Gruppenmodelle und Werkzeuge zur Verwaltung der Gruppen.

Über jeden Benutzer werden allgemeine und kursspezifische Daten erfasst. Bei den allgemeinen Daten handelt es sich um kursübergeordnete, persönliche und leistungsbezogene Daten (vgl. Schneider 2004, 157f).

Über die Administrationswerkzeuge werden alle Aktivitäten der Benutzer protokolliert, dazu gehören die Anmeldedaten (genauer Zeitpunkt des Beginnes und des Endes einer Sitzung, IP-Adresse der Benutzer) und die Aktivitäten, wie Aufruf der einzelnen Seiten und die dazugehörenden Aktionen wie up- bzw. download von Files (vgl. Abbildung 1).

\section{Thema 2}

国 Arbeitsmaterial: Datenmodellierung

3 Ansichten - neueste Dienstag, 31 Oktober 2006, 07:43

Aufgabe: Hausübung 2

Zuletzt geändert: Dienstag, 31 Oktober 2006, 07:46 (7 Tage ⿶055 er-2.doc

Noch nicht bewertet

Abbildung 1: Aktivitäten eines Benutzers zu einem Thema 


\subsubsection{Evaluationswerkzeuge}

Um die Lernerfolge der Studierenden zu ermitteln, integrieren die einzelnen Lernplattformen eine Reihe von Werkzeugen zur Evaluierung (Assessment). Die Beurteilungsverfahren bewerten die Fähigkeiten und Fertigkeiten sowie den Wissensstand der Lernenden. Die Assessment Tools umfassen Werkzeuge für die Generierung, Auswertung und Verwaltung von Online-Fragebögen, Quizzes und Tests (Baumgartner et al. 2004, 113). Die Lernenden können beispielsweise am Ende einer Kurseinheit einen Test absolvieren, um Auskünfte über ihren Lernerfolg und gegebenenfalls auch Anregungen für den weiteren Lernprozess zu erhalten.

Für Prüfungen steht den Lehrenden eine Reihe von Tests zur Verfügung, die üblicherweise eine Form von Multiple Choice-Fragebögen sind. Für die Bewertung von schriftlichen Arbeiten können die Lehrenden eine Reihe weiterer Werkzeuge verwenden, wie Überprüfung des Abgabezeitpunktes und eine Bewertungs- und Rückmeldemöglichkeit.

Abbildung 2 zeigt die Auswertung aus einem Assessmentwerkzeug. Die Testergebnisse einer Lehrveranstaltung werden in Beziehung zu einer Testreihe mit mehreren (in diesem Fall 15) Lehrveranstaltungen gesetzt. So ist ersichtlich, ob die Ergebnisse der Lernenden einer Lehrveranstaltung unter oder über den Ergebnissen von Parallelveranstaltungen liegen. Die Interpretation dieser Auswertung ist dann in alle Richtungen möglich, sowohl die Lernenden betreffend als auch die Lehrenden.

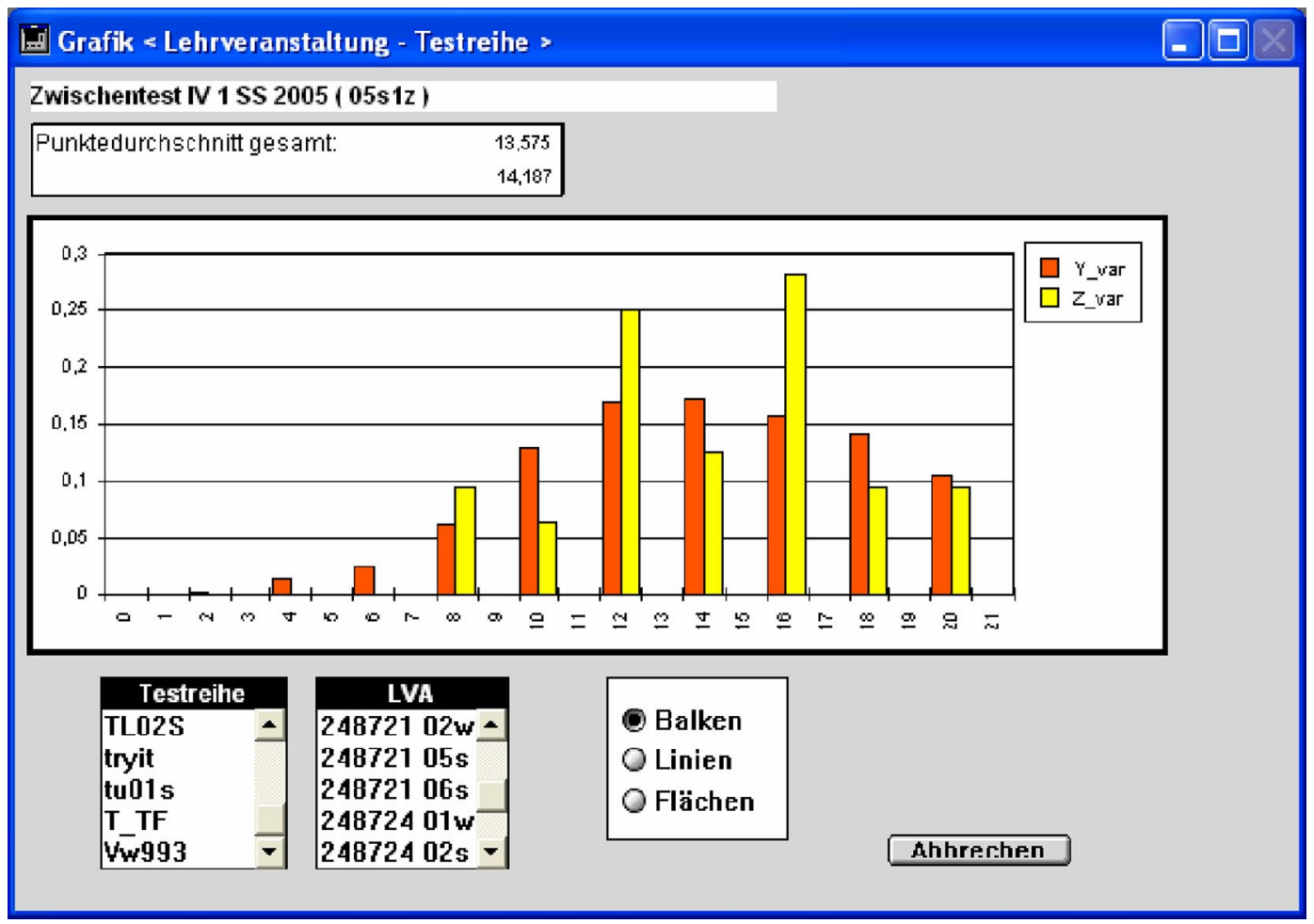

Abbildung 2: Assessmentwerkzeug: Testergebnis einer Lehrveranstaltung im Vergleich einer Testreihe 


\subsubsection{Autorenwerkzeuge und Darstellung von Inhalten}

Die Distribution von Lernmaterial ist eine der Kernaufgaben jeder Lernplattform. Die Lernmaterialien liegen in unterschiedlicher Form und Medien vor bzw. werden in der Lernplattform erstellt. Für die Lehrenden steht eine Reihe von Werkzeugen zur Verfügung, die die Erstellung von Lernmaterialien unterstützen. Die einzelnen Lernplattformen unterscheiden sich in diesem Werkzeugbereich sehr stark. Eine Reihe von Lernplattformen ist aus Systemen hervorgegangen, die ursprünglich als Autorenwerkzeug zur Kurserstellung gedacht waren und denen im Laufe der Zeit Kommunikations- und Kooperationswerkzeuge hinzugefügt wurden. Klassische Autorensysteme kommen speziell bei der multimedialen und didaktischen Aufbereitung von Lerninhalten zum Einsatz. Sie unterstützen die Entwicklung von Lernsystemen ohne dass in konventionaler Weise programmiert werden muss (vgl. Seufert 2005, 5.0-13). Zu den Autorenwerkzeugen gehören auch Kursvorlagen, die die Grundstruktur von Kursen vorgeben und so die Arbeit der Lehrenden erleichtert.

Andere Lernplattformen unterstützen die Lehrenden bei der Erstellung von Lernunterlagen eher wenig. Sie bieten aber den Lehrenden ein umfangreiches Instrumentarium zur Verwaltung von Lernmaterialen, die in anderen Anwendungsprogrammen erstellt wurden.

\subsubsection{Weitere Werkzeuge}

In den Lernplattformen finden sich noch eine Reihe weiterer Werkzeuge wie: Suchfunktionen nach Lerninhalten oder sonstigen Informationen wie z. B. Benutzer, Kalenderfunktion mit Benachrichtigung als persönlicher Kalender bzw. auch als Gruppenkalender, Bookmarks zur Erleichterung der Navigation, Annotationen und Offlinebearbeitung bei der Kurserstellung und bei der Bearbeitung des Kursmaterials. Die Werkzeuge stehen sowohl den Lehrenden und Administratoren als auch den Lernenden zur Verfügung. 


\section{Privatsphäre}

Freiheit und Schutz von Information und Kommunikation sind wichtige Dimensionen des Persönlichkeitsschutzes von Menschen, es ist ein auf Verfassungsebene geschütztes Grundrecht. Die Entwicklung der Informations- und Kommunikationstechnologien durchdringt alle Lebensbereiche, so auch den Bereich des Lernens. Die technischen Entwicklungen bringen neben den erwünschten Wirkungen, wie beispielsweise die Überbrückung von Zeit und Raum, auch eine Reihe von unerwünschten Nebenwirkungen, wie das ungeheure Kontrollpotential, das der Informations- und Kommunikationstechnik innewohnt (vgl. Peissl 2003, 155).

Der Schutz der Privatsphäre umfasst nicht nur das „Recht, in Ruhe gelassen zu werden“, sondern auch das aktive Recht einer Person, darüber zu bestimmen, welche seiner Daten von anderen genutzt werden und in welchem Umfang und unter welchen Bedingungen diese dieses Wissen verwenden dürfen. Entscheidend für Autonomie ist dies deshalb, weil man sich nur dann gegenüber Anderen selbstbestimmt verhalten kann, wenn man weiß, was diese über einen selber wissen (vgl. Kuhlen 1999). Im Zuge der Diskussion der Datenschutzgesetze wurden Mindeststandards bei der Datenerhebung und Datenverarbeitung in Form von „Fair Information Practices“ (www.privacyrights.org) bzw. der „Empfehlung des Rates über Leitlinien für den Schutz des Persönlichkeitsbereiches und den grenzüberschreitenden Verkehr personenbezogener Daten“ (OECD 1980) festgelegt und als acht Grundprinzipien formuliert (Langheinrich 2005, 334f):

1. Beschränkung der Datenbeschaffung (collection limitation): Daten sollten in rechtmäßiger Weise und wenn immer möglich mit der Einwilligung des Datensubjekts erhoben werden.

2. Qualität der Daten (data quality): Die erhobenen Daten sollten dem Zwecke ihrer Erhebung angemessen, korrekt, vollständig und aktuell sein.

3. Zweckbestimmung (purpose specification): Der Zweck der Datenerhebung sollte vorher festgelegt werden.

4. Limitierte Nutzung (use limitation): Zu einem bestimmten Zweck gesammelte Daten sollten nicht für andere Zwecke genutzt werden.

5. Sicherheit der Daten (security): Die gesammelten Daten sollten adäquat vor Verlust, Diebstahl oder unerlaubten Änderungen geschützt werden.

6. Transparenz (openness): Die Methoden der Datenverarbeitung sollten offen gelegt werden.

7. Beteiligung (individual participation): Dem Einzelnen sollte ein gebührenfreies Recht auf Auskunft sowie die Richtigstellung und Löschung seiner Daten zustehen.

8. Verantwortbarkeit (accountability): Die für die Datenverarbeitung Verantwortlichen sollten für Verstöße zur Rechenschaft gezogen werden können.

Das Konzept der informationellen Selbstbestimmung (es gibt dem Einzelnen das Recht, selbst über die Preisgabe und Verwendung seiner personenbezogenen Daten zu bestimmen) erweitert die Fair Information Practices um einen partizipativen Ansatz. „Die informationelle Selbstbestimmung schützt einmal die selbstbestimmte Entwicklung und Entfaltung des Einzelnen. Diese kann nur in einer für ihn kontrollierbaren Selbstdarstellung und Rückspiegelung durch die Kommunikation mit anderen gelingen (Roßnagel 2005, 463). Das deutsche Bundesverfassungsgericht legte in seiner Entscheidung zur Volkszählung fest „Individuelle Selbstbestimmung setzt aber - auch unter den Bedingungen moderner Informationsverarbeitungstechnologien - voraus, daß dem Einzelnen Entscheidungsfreiheit über vorzunehmende oder zu unterlassende Handlungen einschließlich der Möglichkeit gegeben ist, sich auch entsprechend dieser Entscheidung tatsächlich zu verhalten. Wer nicht mit hinreichender Sicherheit überschauen kann, welche ihn betreffende Informationen in bestimmten Bereichen seiner sozialen Umwelt bekannt sind, und wer das Wissen möglicher Kom- 
munikationspartner nicht einigermaßen abzuschätzen vermag, kann in seiner Freiheit wesentlich gehemmt werden, aus eigener Selbstbestimmung zu planen oder zu entscheiden“ (Bundesverfassungsgericht 1983).

Die informationelle Selbstbestimmung ist im Volkszählungsurteil aus der Menschenwürde und dem Persönlichkeitsschutz abgeleitet worden, gewinnt ihre Bedeutung aber in dem aus den Kommunikationsgrundrechten abgeleiteten Freiheitsrecht. Sie zielt auf eine Kommunikationsordnung, die einen selbstbestimmten Informationsaustausch ermöglicht.

Dem Entwickler und Betreiber von Informationssystemen, hier im speziellen Fall einer Lernumgebung, obliegt die Entscheidung, welche personenbezogenen Daten erfasst und verarbeitet werden. Es stellt sich die Frage, von wem die Daten verarbeitet werden und welche Auswirkungen das auf die Betroffenen hat (vgl. Karat 2005, 154).

Die Gefährdung der Privatsphäre wird sowohl durch die technische Entwicklung der Systeme als auch durch sozioökonomische Veränderungen hervorgerufen. Die technischen Überwachungsmöglichkeiten beinhalten ein Kontrollpotential, das oft unbemerkt wirkt (vgl. Peissl 2003, 157).

Gerade im Zusammenhang von Lernplattformen wird das Überwachungspotential von den Beteiligten, vor allem auf Seiten der Lernenden, kaum registriert. Das Problembewusstsein ist bei den Studierenden wenig ausgeprägt, weil sie einerseits über die Überwachung nicht Bescheid wissen. Auf der anderen Seite stehen die Beteiligten nicht vor der bewussten Entscheidung, welche Daten sie bekannt geben möchten. Allein durch die Benutzung der Lernplattform werden ihre personenbezogenen Daten gespeichert. Die Frage im Sinne der informationellen Selbstbestimmung, ob sie ihre personenbezogenen Daten in der Lernplattform preisgeben wollen, stellt sich somit für die Lernenden gar nicht. Wenn sie an einem entsprechenden Kursangebot teilnehmen wollen, müssen sie mit der Lernplattform arbeiten und dort ihre Datenspuren hinterlassen. 


\section{Identitätsinfrastruktur}

Die Lehrenden und die Lernenden authentifizieren sich in der Lernplattform. Das Identitätsmanagement befasst sich mit der Verwaltung der Benutzerdaten. Eine Abbildung der gesamten Identität einer realen Person in eine digitale Identität in der digitalen Welt ist nicht möglich. Wichtige Teile einer Identität, wie Name, E-Mail-Adresse, Studienrichtung können in der digitalen Identität abgelegt werden. Wie wir auch in der realen Welt verschiedene Rollen einnehmen, einmal als Freundin, ein anderes Mal als Arbeitskollegin und unterschiedliche Informationen von uns preisgeben, so können auch verschiedene digitale Identitäten für unterschiedliche Anwendungen angelegt werden.

Die Identität einer Person kann in verschiedene Teilaspekte getrennt werden:

\section{I Anonymität}

Bei einer leeren Identität handelt es sich um eine Identität, die keinerlei Attribute hat und somit praktisch anonym ist. Ein anonymer Benutzer tritt mit keinerlei Identitätsattributen gegenüber einem Dienst auf. Dies ist eine sinnvolle Methode für den Schutz von personenbezogenen Daten, denn es werden keinerlei Daten zur Person erfasst. Auf technischer Ebene bietet das Internet keine absolute Anonymität. Bei jedem Zugriff auf einen entfernten Rechner gibt der Rechner des Benutzers zumindest die IP-Adresse bekannt, damit er auch Daten vom entfernten Rechner erhalten kann. Mit Hilfe der IP-Adresse und des Zeitpunktes der Nutzung dieser Adresse ist es für den Provider einfach, den Rechner dieser Nutzung zu identifizieren.

In der Zwischenzeit gibt es eine Reihe von Projekten, die sich damit beschäftigen, das Internet zu anonymisieren bzw. anonymisierende Techniken zu verwenden. Ein Beispiel dafür ist das JAPSystem, bei dem die Anfragen eines Benutzers über mehrere unabhängige Knoten geleitet werden und damit die IP-Adresse des Absenders versteckt (Berthold et al. 2004).

In Lernplattformen ist der Zugang zu einem beschränkten Teil meist in Form eines „Gast“-Zuganges möglich. Dieser anonymen Identität steht eine sehr eingeschränkte Funktionalität zur Verfügung. Die Teile, die anonym eingesehen werden können, müssen dezidiert freigegeben werden. Der Zugang zu einem bestimmten Kurs ist über einen anonymen Benutzer meist nicht möglich.

\subsection{Pseudoidentität}

Eine Pseudoidentität ist eine von einer Person selbst gewählte oder von einer anderen Person zugewiesene Repräsentation. Die Person nutzt ein Pseudonym, um sich selbst zu präsentieren. Die Pseudoidentität spiegelt eine Teilidentität wider, die meist ein Interessensgebiet oder ein Wunschbild der Person beinhaltet. Pseudoidentitäten kennt man vor allem aus Chat-Rooms, Onlinespielen, Diskussionsforen usw. Die Identifizierung von Kommunikationsteilnehmern über Pseudonyme ist eine gängige Praxis im Web. Dabei besteht die Möglichkeit, das Pseudonym zu wechseln oder gegenüber verschiedenen Partnern unterschiedliche Pseudonyme zu verwenden (Berthold et al. 2004). Dem Pseudonym werden Ressourcen zur Nutzung zugeordnet, das Pseudonym selbst ist dabei identifizierbarer Schlüssel. 
Es gibt eine Reihe von Abstufungen bei Pseudonymen, dabei steht am einen Ende die Anonymität des Benutzers und am anderen die Identifizierung. Zwischen beiden Extremen liegen mehrere Stufen der Pseudonymität. Je näher das Pseudonym der realen Identität kommt, umso höher ist das erforderliche Schutzniveau für personenbezogene Daten.

In Lernplattformen wird normalerweise nicht mit Pseudonymen gearbeitet. Um eine genaue $\mathrm{Zu}-$ ordnung der Aktivitäten zu ermöglichen, müssen sich die Benutzer beim Einstieg identifizieren.

\subsection{Persönliche Identität}

Eine persönliche Identität enthält alle Attribute einer realen Identität, sie besteht also aus persönlichen Daten jeder Art. Sie ist gesetzlich durch das Datenschutzgesetz geschützt.

Die Menge aus Name und E-Mail-Adresse bildet eine eigenständige digitale Identität. Die digitale Identität ist eine Abbildung einer Person in elektronischen Medien, die nur bedingt reversibel und damit nicht eindeutig ist. Jede digitale Identität ist durch eine Menge von Identitätsattributen beschrieben.

Die Identifizierungsmechanismen in Lernplattformen laufen in der Regel über die Angabe von Benutzernamen und Passwort. Als zusätzliche Attribute werden E-Mail-Adresse und IP-Adresse erfasst. Die Zuordnung zu bestimmten Gruppen (wie beispielsweise Lehrveranstaltungen) wird der Identität zugewiesen und ist im jeweiligen Benutzerprofil ersichtlich.

Über Cookies wird die Interaktion zwischen Server und Web-Client teilweise automatisiert. Ein Cookie ist eine kleine Textinformation, die der Browser im Auftrag eines bestimmten WebserverHosts speichert und beim nächsten Besuch bei diesem Server wieder zurückliefert (Kerksen 2003, 960). Der Cookie-File speichert Informationen über die Benutzeridentität, letzte Aktivitäten in der Web-Site oder Passwortinformationen. Die Cookie-Technologie birgt eine Reihe von Problemen im Zusammenhang mit der Privatsphäre (Jerman-Blažič 2005):

- Sicherheitsrisiko: Sensible Informationen werden in Cookies gespeichert und können offen über das Internet weitergegeben werden. Der Inhalt der Cookies kann, zumindest theoretisch, von jedem abgefangen werden bzw. von Backdoors ${ }^{2}$ verwendet werden. In Umgebungen, in denen mehrere Nutzer den selben Rechner teilen, etwa in Laborräumen, besteht gegebenenfalls die Gefahr, dass ein noch gültiger Cookie vom nächsten Nutzer des Rechners verwendet wird und dieser mit den Einstellungen des vorhergehenden Benutzers arbeitet.

- Monitoring: Die Benutzeridentifizierung über Cookies kann auch als Einbruch in die Privatsphäre gesehen werden. Beispielsweise kann ein physischer Shop anonym betreten werden, die einzelnen Aktivitäten werden nicht registriert. Anders verhält es sich in einem Online-Shop, wo über Cookies jeder Benutzer identifiziert und die Aktivitäten registriert werden. Es wird daher die Forderung abgeleitet, auch eine Lernplattform wahlweise personifiziert oder anonym betreten zu können.

- Datenweitergabe: Personenbezogene Daten, die in Cookies abgelegt sind, können über Datenaustausch von anderen Sites (z. B. Ausbildungspartner, mit denen zusammengearbeitet wird) verwendet werden. ,This sharing of data may extend as far as cookies being synchronised for a group of educational activities. This implies that personal information supplied voluntarily at

2 Backdoors sind Schadprogramme, die Dritten den Zugang unter Umgehung der Zugriffssicherung zu einem Computer ermöglichen. 
one site may be used to track or identify an individual at other sites where they have never intentionally disclosed such information“ (Jerman-Blažič et al. 2005, 563).

- Begrenzte Kontrolle: In den neueren Webbrowsern können die Studierenden den Inhalt und die Verwendung der Cookies kontrollieren. Für die meisten Benutzer handelt es sich aber dabei eher um eine unsichtbare Technologie.

- Datensammlung: Eine andere Art, wie Cookies zum Sammeln von personenbezogenen Daten verwendet werden, sind so genannte Web-Bugs. Als Web-Bugs (Web-Wanzen) bezeichnet man kleine Grafiken in HTML-E-Mails oder auf Webseiten, die eine Logfile-Aufzeichnung und eine Logfile-Analyse ermöglichen. Die meist nur $1 \times 1$ Pixel kleinen Bilder sind häufig auch transparent oder in der Farbe des Hintergrunds, damit sie nicht auffallen. Wird ein Dokument geöffnet, dann wird dieses kleine Bild von einem Server im Internet geladen, wobei dieser Download dort registriert wird. So kann der Betreiber des Servers sehen, wann und wie viele Nutzer diesen Web-Bug verwenden, bzw. ob und wann eine E-Mail geöffnet oder eine Webseite besucht wurde.

\subsection{Identitätsmanagement}

Das Identitätsmanagement einer Lernplattform übernimmt die Benutzerverwaltung. Über die Benutzerverwaltung wird auch die Zuteilung der Rollen verwaltet, wie beispielsweise Lehrende, Studierende oder Tutor. Mit der Zuteilung von Rollen werden auch Rechte und Ressourcen definiert (vgl. Abbildung 3).

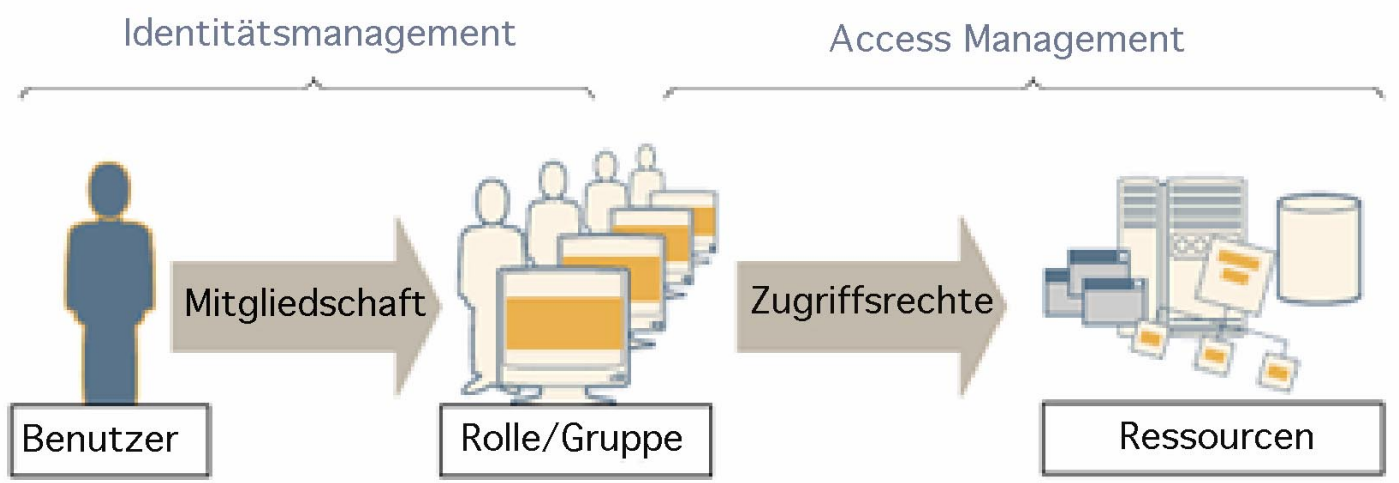

Abbildung 3: Identitätsmanagement (Identity- und Accessmanagement 2006)

Die Administration der Lernplattform legt fest, welche Attribute einer Identität verwendet werden. Die Attribute können auch aus anderen Systemen übernommen werden, wie beispielsweise der Studienadministration. Jedem Benutzer steht ein Identitätsmanager zur Verfügung, in dem er selbständig entscheidet, wann welche Informationen über ihn herausgegeben werden. Der Erstellung dieses Profils kommt besondere Bedeutung zu, weil in der Lernplattform über die digitale Identität kommuniziert wird. 


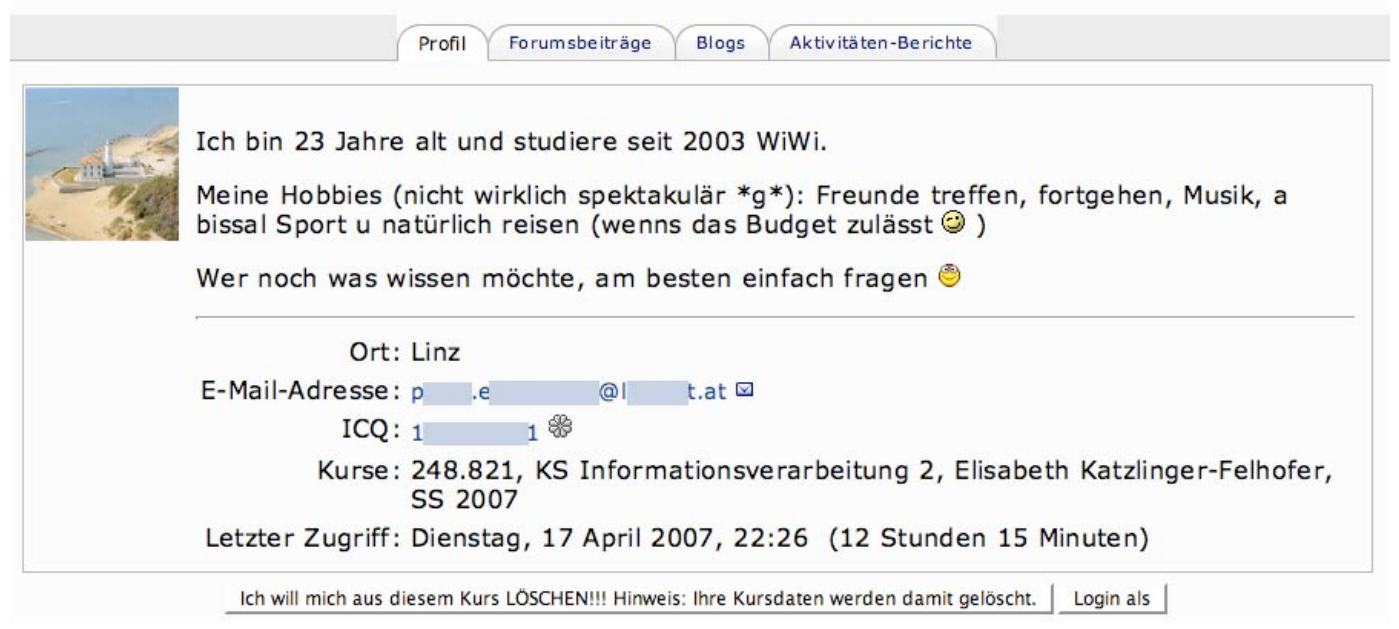

Abbildung 4: Benutzerprofil Studierende (aus Moodle)

Abbildung 4 zeigt einen Auszug aus dem Benutzerprofil von Studierenden in der Lernplattform Moodle. Die persönlichen Angaben werden von den Studierenden gemacht, die Informationen über die belegten Kurse und den letzten Zugriff werden vom System ergänzt.

Das Profil kann über den Identitätsmanager um benutzerdefinierte Kategorien und Attribute erweitert werden. Das adaptierte Profil steht den potentiellen Kommunikationspartnern innerhalb der Lernplattform zur Verfügung. Es können nicht für verschiedene Gruppen, wie beispielsweise einzelne Lehrveranstaltungen, unterschiedliche persönliche Profile erstellt werden. Den Benutzern muss aber klar sein, dass das über den Identitätsmanager geänderte Profil den einzelnen Kommunikationspartnern (wie allen Studierenden aus einer Lehrveranstaltung, Lehrende verschiedener Lehrveranstaltungen) zur Verfügung steht. Lernplattformen zeichnen sich unter anderem auch dadurch aus, dass sie von unterschiedlichen Benutzergruppen verwendet werden. In der Lernplattform meldet sich jeder mit einer Identität (und damit mit einem Benutzerprofil) an. Mit diesem einen Profil wird mit allen anderen kommuniziert.

Jede Person ist meist Mitglied nicht nur in einer sondern in mehreren Gruppen. Innerhalb jeder Gruppe können sich unterschiedliche Kommunikationsstile entwickeln, von sehr informell bis sehr formell. Über das Profil können alle Gruppenmitglieder dieselben Informationen über eine Person einsehen. Das bereitet oft Schwierigkeiten, wenn beispielsweise eine Person ihr Profil im Identitätsmanager für eine informelle Gruppe einrichtet, diese Person aber dann Mitglied in anderen (eher formellen) Gruppen wird und das Profil nicht entsprechend anpasst. 


\subsection{Awareness}

Den Mechanismen der gegenseitigen Wahrnehmung (Awareness, Gewärtigkeit) in kooperativen Arbeitsumgebungen kommt eine besondere Bedeutung zu. Ohne detailliert auf das Forschungsfeld der Awareness eingehen zu wollen (Hoffman 2004, 12f), ist als Teil von kooperativen Handlungen die Wahrnehmung der Handlungen der Kooperationspartner zu berücksichtigen. „Gewärtigkeit ist eine Interaktionsform zwischen menschlichen Akteuren und einer Umgebung. Gewärtigkeit wird zum einen durch die Eigenschaften und Verhaltensweisen einer Umgebung ermöglicht und hängt zum anderen von den Wahrnehmungsfähigkeiten des Individuums ab. Auch eine elektronische Umgebung sollte das Geschehen, d. h. auf sie wirkende Veränderungen und deren Ursachen für die Akteure, wahrnehmbar machen. Die Umgebung produziert dazu GI (Gewärtigkeitsinformation Anm.) über die Anwesenheit von Personen sowie über deren Aktionen.... Gewärtigkeit ist erforderlich, damit ein Individuum situiert handeln kann“ (Pankoke-Babatz et al. 2004, 272).

Die Formen der Awareness reichen von der Rückmeldung der Anwesenheit der Kooperationspartner bis zur detaillierten Übermittlung bestimmter Handlungen innerhalb des gemeinsamen Handlungsbereiches (Hampel et al. 2004). Das Geschehen sollte kurz- und mittelfristig dokumentiert werden. Awareness-Unterstützung sollte umgebungsspezifisch, antizipierbar konstant und reziprok sein, dadurch lassen sich die Gefahren der Informationsüberlastung und der Verletzung der Privatsphäre minimieren (vgl. Pankoke-Babatz et al. 2004, 272).

Für die Unterstützung des Gruppengefüges gibt es mehrere Möglichkeiten, die Kommunikationsbeziehungen $\mathrm{zu}$ visualisieren. In Abbildung 4 ist als Beispiel aus der Lernplattform Moodle die Liste aller Personen, die gerade online sind, angeführt. Die Liste bezieht sich auf alle Personen die diese Lernplattform gerade benutzen und nicht nur auf Personen der eigenen Gruppe oder Kurses.

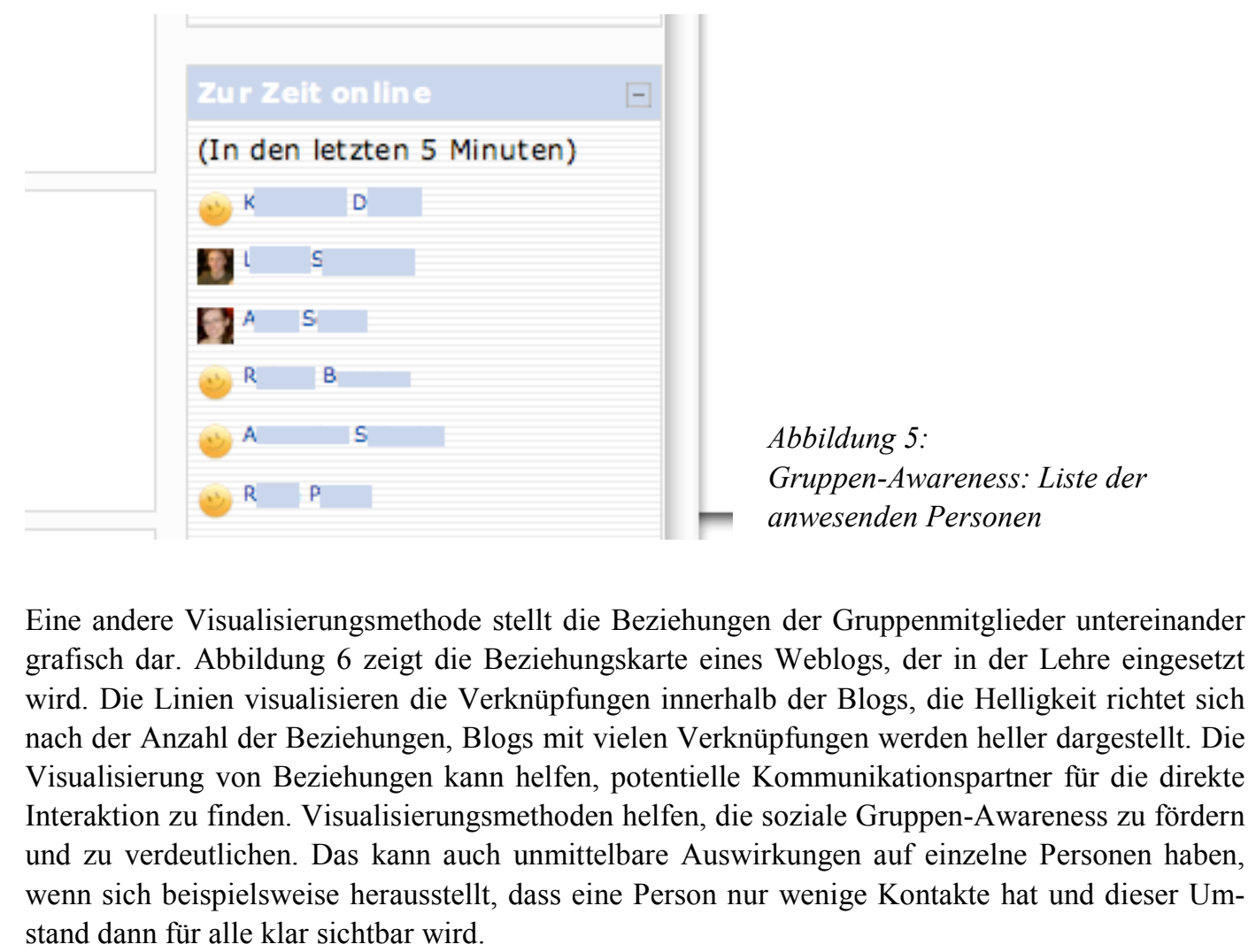




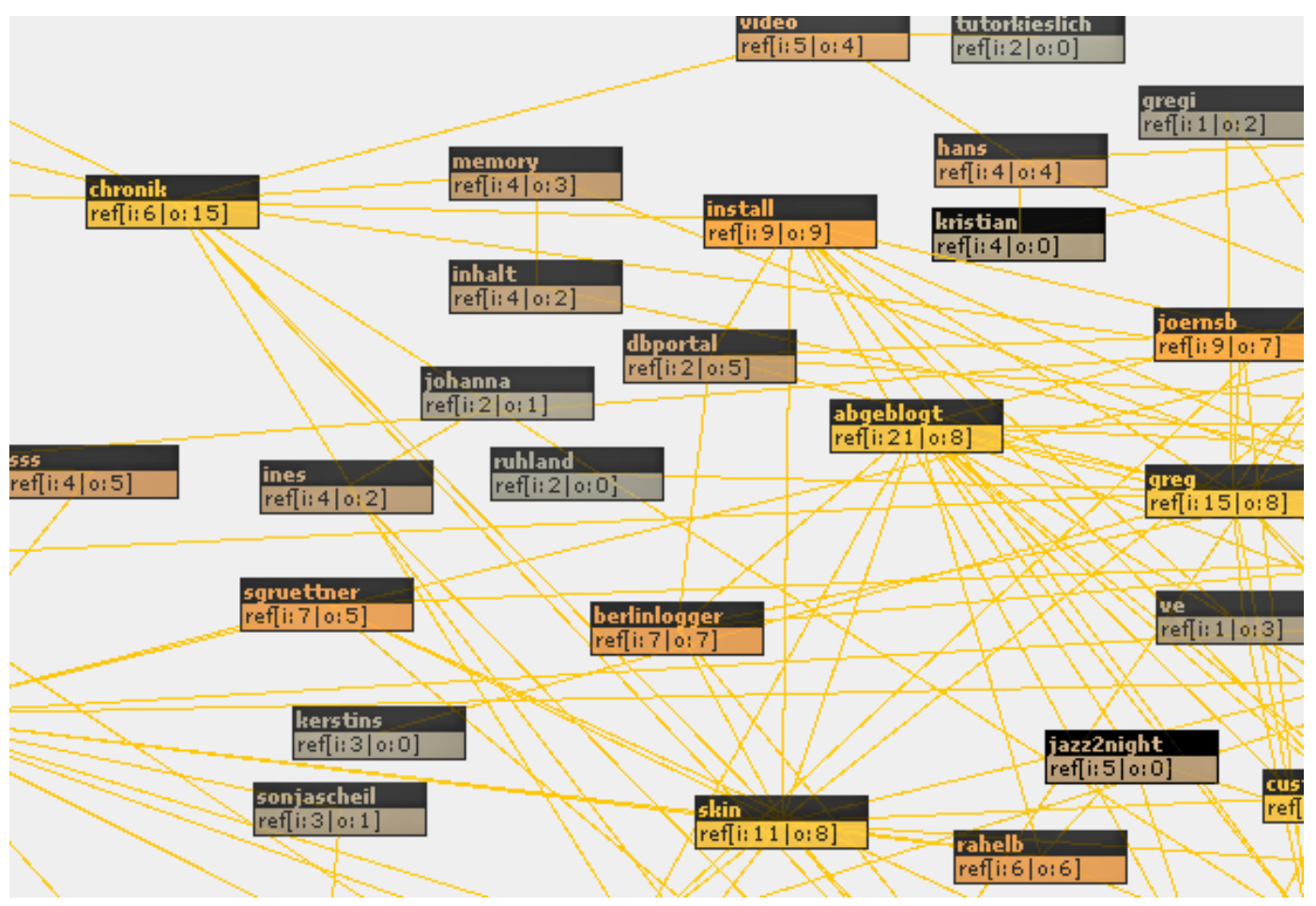

Abbildung 6: Beziehungen innerhalb eines Lehre-Weblogs

Lernplattformen bieten Funktionen, die es erlauben, Lernende im virtuellen Raum zu vernetzen. Ähnliche oder sich ergänzende Fähigkeiten, Interessen und Bedürfnisse können so Bezugspunkte für einen Austausch werden. Dazu müssen personenbezogene Daten der Lernenden erfasst, modelliert und evaluiert werden. Die Profile, die zum Vergleich herangezogen werden, werden auf der einen Seite aus Daten erstellt, die von den Benutzern angegeben werden (Profile Matching). Es handelt sich hier um statische Daten, bei denen oft das Problem der mangelnden Aktualität besteht. Auf der anderen Seite werden die aktuellen Benutzerinteressen und Kenntnisse zum Vergleich verwendet. Diese werden als dynamisch betrachtet, da sie aus den Inhalten der Lernplattform abgeleitet werden, die der Benutzer konsumiert (History Matching) (Reichling et al. 2004).

Innerhalb einer Lerngruppe bilden sich unterschiedliche Expertisen heraus. Für die Lernenden ist es von Interesse, auf das Expertenwissen anderer zugreifen zu können. Das Problem besteht nun darin, wie die einzelnen Lernenden Informationen über die Expertise der einzelnen Gruppenmitglieder erhalten. Mittels „Collaborative Filtering“ wird die Expertise der einzelnen Gruppenmitglieder auf Grund ihres Verhaltens und der Evaluation Ihrer Arbeiten durch die anderen Gruppenmitglieder beurteilt (Aimeur et al. 2006, 239).

Zweig und Webster führten eine Untersuchung über die Akzeptanz von Monitoring-Systemen zur Gruppen-Awareness von geographisch verteilten Arbeitsgruppen durch. Das Monitoring-System stellte den Gruppenmitgliedern die Information bereit, welche Gruppenmitglieder zu einem bestimmten Zeitpunkt interaktionsbereit waren. Bei dieser Untersuchung stellte sich heraus, dass die Akzeptanz von Monitoring-Systemen sehr stark von Persönlichkeitsmerkmalen, wie beispielsweise Extrovertiertheit abhängen. Extrovertierte Personen finden die Monitoring-Systeme fair und hilfreich in ihrer Arbeit, introvertierte Personen sehen darin eine Bedrohung ihrer Privatsphäre (Zweig et al. 2003, 490). Kooperative Lernsituationen sind durchaus mit dem Verhalten von Arbeitsgruppen vergleichbar, sodass diese Ergebnisse auch auf Lernsituationen übertragbar sind. 


\section{Methoden zur Datenauswertung}

\section{I User Profiling}

Das Instrument der Personalisierung von Services und Inhalten ist aus dem E-Business-Bereich seit langem bekannt und dort entwickelt worden. Die Methoden und Konzepte können auch für die Personalisierung von E-Learningangeboten verwendet werden. Angebote und Inhalte können entweder formal (z. B. durch die Anpassung von Schriftgröße, Hintergrundfarbe) oder auch inhaltlich (z. B. Hinweis auf Neuerscheinung in einem bestimmten Fachgebiet) angepasst werden. Personalisierung kann nicht allein von einer Seite aus betrachtet werden (Aehnelt 2003). Es handelt sich dabei um mindest zwei Grundfunktionen:

- Marketingwerkzeug: Angebote und Inhalte, die direkt auf den Verbraucher zugeschnitten sind, lassen sich leichter verkaufen. Moderne Vermarktungsinstrumente, wie beispielsweise Web-basierte elektronische Shop-Systeme, verwenden verschiedenste Personalisierungsinstrumente, um das Angebot möglichst passend dem Kunden zu offerieren. Je mehr Informationen über den Kunden bekannt sind, desto besser können seine Wünsche und Anforderungen berücksichtigt werden.

- Individualisierung: Personalisierung ist auch ein Instrument der Individualisierung, mit dem auf die persönlichen Anforderungen eines Kunden bzw. Nutzers eingegangen werden kann. Als Beispiel dafür können auch moderne Softwarepakete gesehen werden. Nicht jeder User verwendet die gleichen Funktionalitäten eines Systems im gleichen Umfang, es ist möglich, bestimmte Funktionen ein- bzw. auszuschalten. Zum anderen sind Systeme auch in der Lage, aus der Aktionshistorie des Benutzers dynamische Programmmenüs zur Verfügung zu stellen (z. B. Microsoft-Office-Anwendungen).

„Der Nutzen der Personalisierung lässt sich darum aus den angeführten Sichtweisen ersehen. Sie dient dem Hersteller eines Produktes als Argument und Instrument der Vermarktung. Dem Verbraucher eines personalisierbaren Produktes hilft sie hingegen, Arbeitsabläufe effektiver zu gestalten. Beide Sichten zeigen, dass Personalisierung in der modernen Zeit ein gewichtiges Werkzeug ist, um den Erfolg eines Produktes positiv zu beeinflussen, indem letztlich die Wünsche seines Verbrauchers beachtet werden“"(Aehnelt 2003).

Der Personalisierungsprozess lässt sich in drei Phasen unterteilen

- Informationsakquise: Es werden über die Benutzer unterschiedliche Informationen mit verschiedenen Techniken gesammelt.

- Informationsanalyse: Die Informationen werden analysiert und in Zusammenhang mit anderen gesetzt, um daraus bestimmte Schlussfolgerungen zu ziehen.

- Personalisieren: Zuordnung der gewonnenen Informationen zu einem bestimmten Benutzer.

Grundlage jeder Personalisierung ist, möglichst viele Informationen über eine Person zu sammeln. Dabei sind folgende Informationen von Interesse:

- Benutzerinformation: hier werden persönliche Charakteristiken eines Benutzers beschrieben, wie biographische (Name etc.), geographische (Land, Bundesland etc.), soziographische (Alter, Geschlecht, Schulbildung) oder psychographische (Lebensstil, Kaufverhalten, Zahlungsgewohnheiten). Für Lernplattformen sind Informationen über Wissen und Kenntnisse, Fertigkeiten, Interessen und Ausbildungsstand von besonderem Interesse. 
- Benutzungsinformation: Es werden Informationen über alle beobachtbaren Interaktionen gesammelt und der Person zugeordnet. Dazu zählen Selektionsaktionen (von Links, Informationen), zeitliches Betrachtungsverhalten (Betrachtungsverhalten, Sequenzen), Bewertungen, Kaufverhalten usw.; Informationen, die Regelmäßigkeiten in der Benutzung beschreiben, zählen ebenso in diese Kategorie. Es wird versucht, bestimmte Verhaltensmuster zu erkennen.

- Umgebungsinformation: Es werden alle signifikanten Eigenschaften der technologischen und örtlichen Umgebung des Benutzers aufgezeichnet, wie Softwareumgebung (Browser, Betriebssystem), verwendete Hardware (Bandbreite, Rechenleistung) und die Örtlichkeit (wobei die Granularität zwischen dem Staat, in dem sich die Person befindet und den exakten Positionskoordinaten variieren kann) (Kobsa 2004, 300).

Aus dem Umfang der hier angeführten Informationen wird ersichtlich, dass Personalisierung auf unterschiedlichen Interaktionsebenen stattfinden kann. Alle gewonnenen Informationen werden in einem Benutzerprofil gespeichert.

Für die inhaltliche Personalisierung können zwei Technologiearten unterschieden werden (Aehnelt 2003):

- Pull-Technologie: Sie repräsentieren benutzergetriebene Informationen, dabei erweitert der Benutzer sein Profil um Informationen.

- Push-Technologie: Sie sind durch systemgetriebene Informationen charakterisiert. Die im Benutzerprofil gespeicherten Informationen werden durch verschiedene Methoden ausgewertet. Wenn-Dann-Regeln filtern bekannte oder angenommene Charakteristika des Benutzers heraus. Die Suchwege werden durch die Auswahlhäufigkeit von Inhalten verkürzt. Das Interaktionsverhalten des Benutzers wird beobachtet und analysiert, um seine Interessen herauszufinden. Weiters werden auch gruppenspezifische Charakteristika analysiert, um daraus auf Interessen des Einzelnen zu schließen.

Im E-Learning hat man sich mit der Technik der Personalisierung ebenfalls auseinandergesetzt. Vor dem Hintergrund des selbstgesteuerten Lernens besteht die Forderung nach einem möglichst individuell gestalteten Lernprozess. Beim selbstgesteuertem Lernen bestimmt der Lernende einerseits Zeit und Ort des Lernens, aber auch den Weg durch die Lerninhalte. Struktur und Lerninhalte werden den individuellen Bedürfnissen des Lernenden angepasst, wie beispielsweise die Anpassung der Lerninhalte an das Wissen des Lernenden. Mit Hilfe der Personalisierung ist diese enge Verzahnung möglich.

Das Konzept der Personalisierung ist nur dann in Lernumgebungen realisierbar, wenn Charakteristika der Benutzer bekannt sind. Mit Hilfe der Pulltechnologien kommt es zu einer Auswertung der Interaktion von Lernenden mit dem Lehrinhalt, um die fehlenden Inhalte zu erfassen.

Für das Problem der Personalisierung wurden in den einzelnen Lernplattformen individuelle Lösungen geschaffen, die für andere Lernumgebungen nicht verwendbar ist. Mit Hilfe der Standardisierungen wird versucht, einen Austausch zwischen den Lernumgebungen zu ermöglichen. Ausgetauscht werden Attribute wie Login-Name, bevorzugte Sprache, Lautstärkenpräferenz, Textpräferenz, Arbeitsgeschwindigkeit. Die Frage, die sich in diesem Zusammenhang allerdings stellt, ist, ob dies im Sinne der Privatsphäre und des Datenschutzes wünschenswert ist.

Folgende Bereiche von E-Learning-Angeboten können personalisiert werden (Aehnelt 2003):

- Interaktion des Lernenden mit der Lernumgebung: So können Arbeitsanweisungen unterschiedlich gestaltet werden, von formal knapp bis ausführlich und sehr kollegial. Bei der Personalisierung können auch Umgebungsparameter berücksichtigt werden wie beispielsweise Arbeitsumgebung oder Freizeit. 
- Personalisierung des Umfanges: Um eine Überforderung der Lernenden zu verhindern, ist es möglich, je nach Kenntnisstand den Umfang der Lerninhalte anzupassen. Wenn der Lernende mangelnde Kenntnisse in einem bestimmten Gebiet hat, werden ihm zusätzlich Informationen angeboten.

- Personalisierung der Präsentation: Hier werden die Gewohnheiten des Lernenden bei der Informationsaufnahme berücksichtigt. Angefangen von Textgröße und Lautstärke bis hin zu Vorlieben wie die Aneignung von Lerninhalten über Texte, Animationen oder Video. Gleiches gilt auch für die Arbeitsgeschwindigkeit.

\subsection{User Tracking}

Unter User Tracking versteht man die Aufzeichnung von Benutzeraktivitäten, um diese nachvollziehbar und auswertbar zu machen. Der Betreiber eines Web-Servers, wie beispielsweise einer Lernplattform, zeichnet die Daten aller Besucher auf. Die Daten stehen für Auswertungen zur Verfügung. Es werden die Verbindungs- und die Zustandsdaten eines Systems in Logfiles aufgezeichnet. Sie dienen in der Regel der technischen Überwachung von Systemen (Auslastung, Optimierung, Fehlererkennung, Sicherheit) und sind dazu unbedingt notwendig. Diese Daten werden aber auch für die Erstellung von Benutzerprofilen verwendet. Problematisch können Logfiles vor allem dann werden, wenn sie über einen längeren (als den technisch notwendigen) Zeitraum aufbewahrt und ausgewertet werden. Eine Kooperation oder Abstimmung mit der beobachteten Person ist nicht Voraussetzung; im Gegenteil, er/sie kann sich dagegen kaum schützen.

Die Logfiles werden zum Teil in standardisierter Form als Textfiles abgelegt und enthalten im ausführlichen Fall: die IP-Adresse (Internet Protocol) des Users, die Zugriffszeit, diverse HTML-Befehle (Post, Get etc.), die URL der aufgerufenen Seite/Datei, die URL der vorhergehenden Seite (Referrer-URL), die Art des Protokolls (beispielsweise http), ein Statuscode-Feld, die Anzahl der übertragenen Bytes, Angaben über den genutzten Browser sowie eine User-ID, wenn sich der Benutzer angemeldet hat (vgl. Hansen 2005, 714f).

Diese Daten sind zwar objektiv nachvollziehbar, da sie automatisiert erfasst werden, jedoch sind sie lediglich deskriptiver Art und bedürfen einer Interpretation, um aussagekräftige Daten zu erhalten. Tatsächlich können die aus den Logfiles generierten Daten fehlerbelastet sein:

- Durch die Nutzung von Proxyservern oder lokalen Caches können unvollständige Protokolldateien auftreten, weil nicht alle Zugriffe aufgezeichnet werden.

- Auch die User-Identifikation gestaltet sich auf Grund der Vergabe dynamischer IPs oder auch der Vergabe derselben IP für mehrere User als nicht einfach, eine eindeutige Identifikation ist nur durch eine Registrierung mit Benutzername und Passwort möglich.

Durch die Identifizierung in den Lernplattformen werden in den Protokolldateien eine Reihe weiterer Daten erfasst. Die Führung und Auswertung der Logfiles erfolgt nicht nur aus technischen Gründen, um den Systembetrieb zu gewährleisten, sondern die Daten werden den Lehrenden als Aktivitätsdaten der einzelnen Studierenden zur Verfügung gestellt. In Abbildung 7 ist die Auswertung aus der Lernplattform Moodle angeführt. 


\begin{tabular}{|c|c|c|c|c|}
\hline Zeit & IP-Adresse & $\begin{array}{l}\text { Vollständige } \\
\text { Name }\end{array}$ & Aktion & Information/en \\
\hline Fr 30 März 2007, 02:02 & 8 $\cdot 6 \cdot 4 \cdot 1$ & EE & $t$ forum user report & $k \quad h$ \\
\hline Fr 30 Mărz 2007, 02:02 & 8.6 .4 .1 & M & $t$ userview & k $h$ \\
\hline Fr 30 März 2007, 02:02 & 8.6 .4 .1 & M & $t$ choice view & Tutoriumseinteilung \\
\hline Fr 30 Mărz 2007, 02:01 & 8.6 .4 .1 & eE & $t$ course view & 248.821, KS Informationsverarbeitung 2, Elisabeth Katzlinger-Felhofer, SS 2007 \\
\hline Do 29 März 2007, 12:22 & 8.6 .4 .1 & M & $t$ assignment view & Hausübung 3 \\
\hline Do 29 März 2007, 12:22 & 8.6 .4 .1 & M & $t$ course view & 248.821, KS Informationsverarbeitung 2, Elisabeth Katzlinger-Felhofer, SS 2007 \\
\hline Do 29 Mărz 2007, 12:02 & 8.6 .4 .1 & eE & $t$ userview & $\mathrm{I} \quad \mathrm{W}$ \\
\hline Do 29 März 2007, 12:02 & $8 \cdot 6 \cdot 4 \cdot 1$ & eE & t choice view & Tutoriumseinteilung \\
\hline Do 29 Mărz 2007, 12:02 & 8.6 .4 .1 & M & t course view & 248.821, KS Informationsverarbeitung 2, Elisabeth Katzlinger-Felhofer, SS 2007 \\
\hline Do 29 März 2007, 01:31 & 8.6 .4 .1 & eE & $t$ assignment view & Hausübung 3 \\
\hline Do 29 März 2007, 01:31 & $8 \cdot 6 \cdot 4 \cdot 1$ & EE & $t$ course view & 248.821, KS Informationsverarbeitung 2, Elisabeth Katzlinger-Felhofer, SS 2007 \\
\hline Mi 28 März 2007, 13:59 & & M & t course view & 248.821, KS Informationsverarbeitung 2, Elisabeth Katzlinger-Felhofer, SS 2007 \\
\hline
\end{tabular}

Abbildung 7: Auszug aus der Studierenden-Statistik im Moodle

\begin{tabular}{|c|c|c|c|c|}
\hline Zeit & IP-Adresse & Vollständiger Name & Aktion & Information/en \\
\hline Di 17 April 2007, 11:54 & $14.7 \cdot 5.3$ & Elisabeth Katzlinger-Felhofer & course view & 248.821, KS Informationsverarbeitung 2, Elisabeth Katzlinger-Felhofer, SS 2007 \\
\hline Di 17 April 2007, 11:54 & $14.7 \cdot 5 \cdot 3$ & Elisabeth Katzlinger-Felhofer & course view & 248.821, KS Informationsverarbeitung 2, Elisabeth Katzlinger-Felhofer, SS 2007 \\
\hline Di 17 April $2007,11: 54$ & 14.7 .5 .3 & Elisabeth Katzlinger-Felhofer & assignment view & Hausübung 3 \\
\hline Di 17 April 2007, 11:54 & $14.7 .5 \cdot 3$ & Elisabeth Katzlinger-Felhofer & course view & 248.821, KS Informationsverarbeitung 2, Elisabeth Katzlinger-Felhofer, SS 2007 \\
\hline So 15 April 2007, 19:32 & 8:1: $\cdot 1 \div \cdot 0$ & Elisabeth Katzlinger-Felhofer & course view & 248.821, KS Informationsverarbeitung 2, Elisabeth Katzlinger-Felhofer, SS 2007 \\
\hline So 15 April 2007, 19:31 & $8.1: .11 .0$ & Elisabeth Katzlinger-Felhofer & resource view & Unterwegs im Web 2.0 \\
\hline So 15 April 2007, 19:31 & $8.1: .1 .0$ & Elisabeth Katzlinger-Felhofer & course add mod & resource 11716 \\
\hline So 15 April 2007, 19:31 & 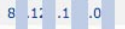 & Elisabeth Katzlinger-Felhofer & resource add & Unterwegs im Web 2.0 \\
\hline So 15 April $2007,19: 29$ & $8.1: .1 .0$ & Elisabeth Katzlinger-Felhofer & course view & 248.821, KS Informationsverarbeitung 2, Elisabeth Katzlinger-Felhofer, SS 2007 \\
\hline So 15 April $2007,19: 28$ & 8.1: .1 .0 & Elisabeth Katzlinger-Felhofer & resource view & Präsentation Web 2.0 - Social Software \\
\hline So 15 April 2007, 19:28 & $\begin{array}{llll}8 & 1: & .1 & .0\end{array}$ & Elisabeth Katzlinger-Felhofer & course add mod & resource 11715 \\
\hline So 15 April 2007, $19: 28$ & $\begin{array}{ll:lll}8 & 1: & .1 & .0\end{array}$ & Elisabeth Katzlinger-Felhofer & resource add & Präsentation Web 2.0 - Social Software \\
\hline So 15 April $2007,19: 28$ & 8.1: .1 .0 & Elisabeth Katzlinger-Felhofer & upload upload & /var/www/apps/content/moodle_data/61 0/Web20.pdf \\
\hline
\end{tabular}

Abbildung 8: Auszug aus Lehrenden-Statistik im Moodle

Nicht nur von den Lernenden, sondern auch von den Lehrenden stehen die Auswertungen der Logfiles zur Verfügung. Abbildung 8 zeigt den Auszug aus der Lehrenden-Statistik. Den Lernenden steht nicht die gesamte Statistik zur Verfügung, sondern nur die letzte Aktivität und die Beiträge in Foren, Wikis und Weblogs.

Die Daten aus den Logfiles können innerhalb der Lernplattform auch grafisch ausgewertet werden. Abbildung 9 zeigt ein Beispiel dazu. Vor allem für Lehrveranstaltungen, die einen interaktiven Charakter haben, können diese Auswertungen Teil der Beurteilungsgrandlage sein.

Es gibt eine Reihe von Untersuchungen, die sich mit den Auswirkungen der elektronischen Überwachung, vor allem von Arbeitnehmern, beschäftigen. Dabei zeigten sich negative Effekte vor allem im Bereich der Produktivität und der moralischen Verantwortung der Arbeitnehmer. So zeigte eine Untersuchung von Ariss, dass nach Selbsteinschätzung der Betroffenen bei drei Viertel der Befragten ihre Arbeitsqualität durch das Monitoring litt (Ariss 2002, 556). Es geht somit darum, eine Balance zwischen betriebsnotwendigen Erfordernissen an Überwachung und Interessen der Betroffenen an Wahrung von Privatsphäre zu finden.

Wenn diese Ergebnisse auf Lernsituationen übertragen werden können, würde das bedeuten, dass die Lernenden ihr Verhalten an die Überwachungssituation anpassen und die Qualität ihrer Leistungen sinken kann. 


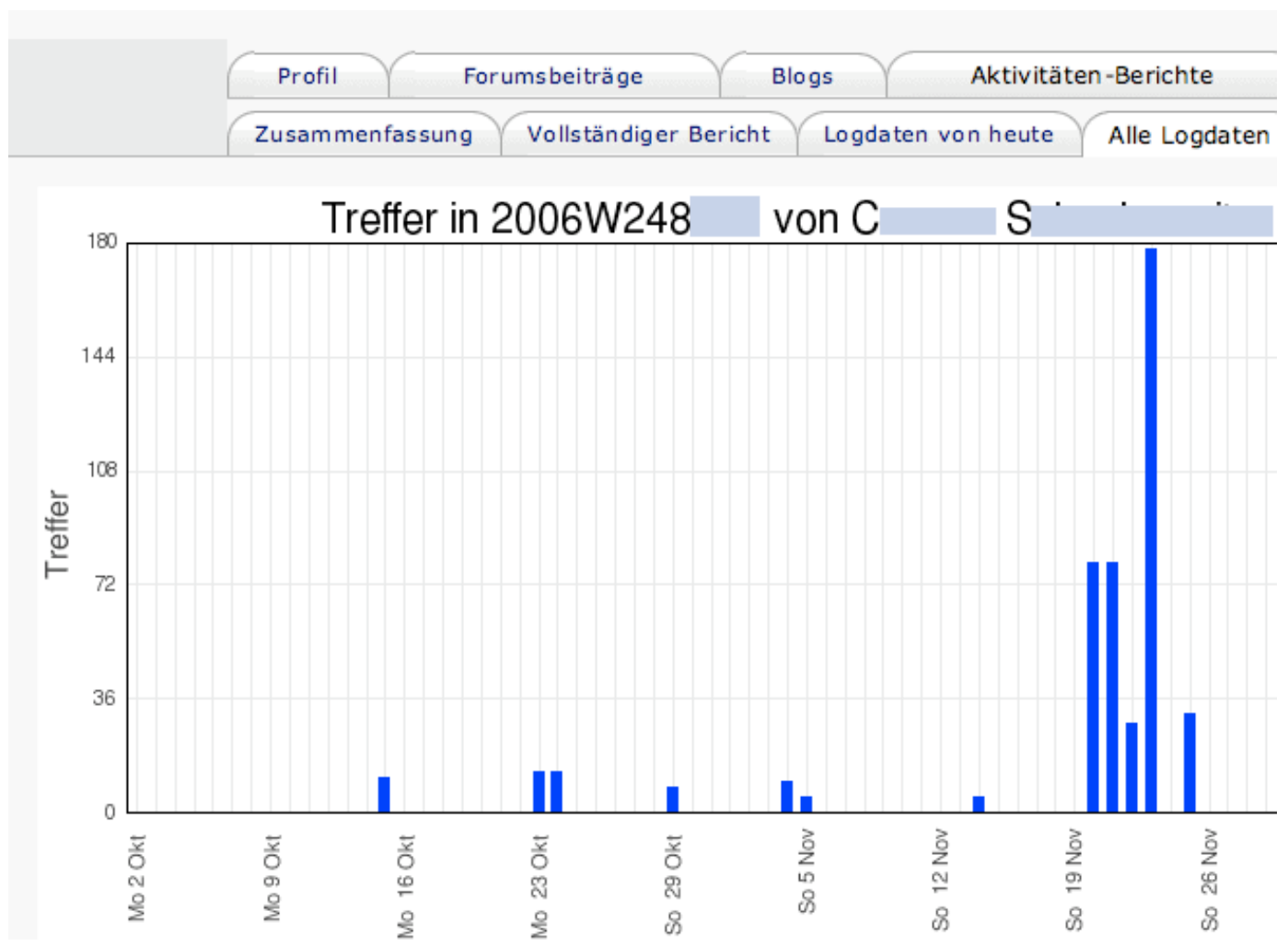

Abbildung 9: Grafische Auswertung der Lernendenaktivitäten

\subsection{Data Mining}

„Als Data Mining (deutsch: Datenbergbau, Daten schürfen) bezeichnet man die softwaregestützte Ermittlung bisher unbekannter Zusammenhänge, Muster und Trends aus dem Datenbestand sehr großer Datenbanken beziehungsweise eines Data Warehouse. Dabei kann der Benutzer bestimmte Ziele vorgeben, für die das System angemessene Beurteilungskriterien ableitet und damit die Datenobjekte der Datenbank(en) analysiert“ (Hansen et al. 2005, 822). Data Mining-Techniken können nicht nur abstrakte Muster oder Trends identifizieren, sondern sie können bei Bedarf auch Detaildaten liefern. Verwendet werden die Erkenntnisse zur Entscheidungsunterstützung und zur Vorhersage von künftigem Verhalten (Laudon et al. 2006, 339).

Jeder Benutzer hinterlässt mehrere Spuren seiner Daten. Mit Hilfe von Data Mining-Techniken können Daten aus verschiedenen Quellen miteinander in Beziehung gesetzt werden und zu einem detaillierten Datenbild (Benutzerprofil) kombiniert werden. Das Bild einer Person, die hinter einer Identität steckt, kann so genauer nachgezeichnet werden, beispielsweise ihre Interessen, Vorlieben oder bevorzugten Arbeitszeiten.

Data Mining verwendet Methoden der Statistik und des maschinellen Lernens. Sie können folgenden drei Kategorien zugeordnet werden (vgl. Hansen et al. 2005, 822):

- Klassifikation: Ein qualitatives Merkmal wird durch eine Reihe von anderen Variablen erklärt. Ein neues Objekt soll so einer bekannten Gruppe zugeordnet werden. Aufgabe der Klassifikation ist es, anhand einer vorgegebenen Trainingsmenge von bekannten Objekten ein Modell auf- 
zubauen, mit dem sich unbekannte Objekte aufgrund ihrer Eigenschaften in Klassen einteilen lassen. Dabei sind sowohl die Klassen als auch die Zugehörigkeit der Trainingsobjekte zu einer Klasse vorher bekannt. Die Attribute können sowohl quantitativ als auch qualitativ sein, das Klassenattribut ist immer qualitativ. Klassifikationsverfahren arbeiten in zwei Phasen. Zuerst wird anhand der Trainingsdaten ein Modell aufgebaut, mit dessen Hilfe man in der zweiten Phase unbekannte Daten, also solche, bei denen das Klassenattribut $\mathrm{c}$ unbekannt ist, in eine passende Klasse einordnet. Ein weiteres wichtiges Ziel der Klassifikation ist es, anhand des aufgebauten Modells Wissen über die Klassen zu erlangen.

- Regression: Dabei wird versucht, ein quantitatives Merkmal durch eine Reihe von anderen Variablen zu erklären. Auch hier dient das gewonnene Modell für Prognosezwecke.

- Segmentierung (Clustering): Um Zusammenhänge in Daten entdecken zu können, ist es hilfreich, gleichartige Daten zu Gruppen zusammenzufassen. Clusteringverfahren leisten dies, indem sie sich ähnelnde Objekte der Eingabedaten in Cluster einteilen. Ein Cluster ist eine Menge von Objekten, die untereinander eine hohe und zu anderen Objekten außerhalb des Clusters eine möglichst geringe Ähnlichkeit aufweisen. Die Ähnlichkeit zweier Objekte wird normalerweise über ihre Distanz angenähert. Ein Verfahren, das bei quantifizierbaren Ausprägungen (wie beispielsweise Alter) gut möglich ist. Bei qualitativen Werten ist die Bildung eines Algorithmus schwieriger.

Anwendungsschwerpunkte von Data Mining finden sich in sehr unterschiedlichen Bereichen wie im E-Business (beispielsweise im Kundenbeziehungsmanagement), in der Medizin (Wirkungen von Medikamenten) oder in der Technik.

Im Zusammenhang mit Lernplattformen ist Text Mining von Interesse, das als spezielle Ausprägung von Data Mining gesehen wird (Hippner et al. 2006, 287). Der Unterschied liegt in der zugrunde liegenden Datenbasis, im Data Mining sind das strukturierte Daten, im Text Mining sind dies unstrukturierte Daten (eine implizite Struktur ergibt sich aus der Grammatik).

„Text Mining umschreibt folglich die Anwendung von Data-Mining-Methoden auf Textdokumente. Die Herausforderung des Text Minings liegt dabei darin, die in einem Text sprachlich wiedergegebene Information für die maschinelle Analyse zu erschließen“ (Hippner et al. 287). Beim Text Mining ist eine zusätzliche linguistische Analyse erforderlich, um die fehlende Datenstruktur zu rekonstruieren. Algorithmen zur linguistischen und zur statistischen Textanalyse werden kombiniert.

Durch die linguistische Analyse erhalten die Texte eine Struktur, es können nun die Verfahren des klassischen Data Minings angewendet werden. „Texte können automatisch vorgegebenen Kategorien zugeordnet werden (Klassifikation) oder sie können so gruppiert werden, dass ähnliche Texte zusammengeführt werden (Segmentierung). Ebenso kann das gemeinsame Auftreten von Termen analysiert werden (Abhängigkeitsanalyse)“ (Hippner et al. 2006, 289).

Anwendungen des Text Minings sind vor allem in Bereichen interessant, in denen viele Dokumente vorliegen und in denen Wissen eine große Rolle spielt. Schriftliche Arbeiten von Lernenden sind ein Beispiel dafür. Durch das Text Mining können die Arbeiten analysiert werden und so eine Grundlage für die Leistungsbeurteilung bilden. Prüfungsarbeiten können nach dem Vorkommen von bestimmten Termen untersucht werden. Durch die Segmentierung können Ähnlichkeiten aufgedeckt werden. 


\section{Resümee}

Es gilt für den Datenschutz und die Wahrung der Privatsphäre in Lernplattformen geeignete Rahmenbedingungen zu schaffen. Informations- und Kommunikationssysteme sind gestaltungsbedürftig und gestaltungsfähig. „Gestaltungsentscheidungen sind immer wieder notwendig etwa bei der Entwicklung technischer Normen, bei der Konzeption der Systeme, bei der Festlegung ihrer Funktionen, bei der Auswahl der Komponenten, bei der Bestimmung von Freiheitsgraden oder bei der Konfigurierung... Bei diesen Gestaltungen entscheiden Informatikerinnen und Informatiker auch immer - bewusst oder unbewusst - über die Chancen und Grenzen informationeller Selbstbestimmung. Datenschutzaspekte sind leicht zu berücksichtigen, wenn dies bei frühen Gestaltungsentscheidungen geschieht, dagegen schwer, wenn dies erst erfolgt, wenn bereits wichtige Strukturentscheidungen getroffen sind, die nachträglich verändert werden müssten“ (Roßnagel 2005, 470).

Das Recht auf Privatsphäre steht der Herausforderung einer technischen und gesellschaftlichen Entwicklung gegenüber, die auch vor dem Bildungsbereich nicht Halt macht. Die Menge der Daten und Informationen und deren Verwendungsmöglichkeiten steigen ständig an. Da die Daten in Lernplattformen einen Personenbezug haben, ist die Privatsphäre betroffen. Durch die Verknüpfbarkeit lässt sich der Personenbezug mit geringem Aufwand herstellen und ermöglicht damit Aussagen über die betroffenen Personen.

Das Spannungsfeld zwischen dem Schutz der persönlichen Freiheit und den Sicherheitsinteressen ist ein zunehmend debattierter Aspekt der Informationsgesellschaft. Die Verarbeitung von personenbezogenen Daten wird für die Betroffenen immer intransparenter, wie auch die Befragung der betroffenen Studierenden gezeigt hat. Dadurch wird eine rationale Bewertung des Interessensausgleichs zwischen Privatsphäre und Sicherheit erschwert.

Mit der fehlenden Transparenz über die verarbeiteten Daten verlieren die Betroffenen die Souveränität über ihre Daten. Mit zunehmender Sensibilität der Daten ist der Verlust dieser Datensouveränität ein Problem. Den betroffenen Lehrenden und Lernenden soll daher offen gelegt werden, welche Daten über sie gespeichert sind und wer Zugriff auf diese Daten hat (z. B. Lehrveranstaltungsleiter bzw. -leiterin, Studienadministration, Systemadministration).

Auch die Aufbereitung der Daten, beispielsweise für die Lehrenden, spielt dabei eine Rolle. Aus der Studierendenbefragung zur Lehrveranstaltung, auf die sich Abbildung 7 bezieht, geht hervor, dass den Studierenden nicht bewusst war, dass der Lehrveranstaltungsleiterin die Auswertung der Aktivitätsdaten in dieser Form vorliegen. Es ist somit in dieser Lernplattform den Lernenden nicht transparent, welche Daten in welcher Form den Lehrenden zur Verfügung stehen.

Es sind nicht nur die Lernenden vom Verlust der Datensouveränität betroffen, sondern auch die Lehrenden in gleichem Maße. Den Systemadministratoren stehen die Logfiles der Lehrenden für Auswertungen zur Verfügung, die im Zuge der Evaluierung der Lehrenden verwendet werden können.

Um das Recht zur informationellen Selbstbestimmung in Anspruch nehmen zu können, sollen die Betroffenen auf Wunsch in die Lage versetzt werden, Datenspuren zu vermeiden. Über das Identitätsmanagement soll auch Anonymität oder Pseudonymität gewährleistet werden. Der Umgang mit Instrumenten des Selbstdatenschutzes erfordert allerdings bei den Beteiligten entsprechende Voraussetzungen, die durch Bildungsangebote vermittelt werden müssen.

Ein Kernbestandteil der informationellen Selbstbestimmung ist die Einwilligung der Betroffenen zur Verarbeitung ihrer personenbezogenen Daten. Mit der Akzeptanz der Nutzungsbestimmungen stimmen die Betroffenen der Verarbeitung zu. Da die Lernplattform ein integraler Bestandteil von Lehrveranstaltungen ist, ist es für die Lernenden nicht möglich, die Zustimmung zur Verarbeitung der personenbezogenen Daten nicht zu geben. 
Datenschutz und Schutz der Privatsphäre sind allerdings nicht die einzigen Interessen, die zu berücksichtigen sind. Sie stehen in Konkurrenz mit anderen wichtigen Interessen, wie beispielsweise dem Sicherheitsinteresse oder der Überprüfbarkeit von Leistungen der Lernenden. Sie verlangen eine Verarbeitung personenbezogener Daten. Es gilt hier einen Ausgleich der konkurrierenden Interessen zu finden.

Für die Lehrenden stehen Daten zur Verfügung, die vor allem die Überprüfung von formalen Anforderungen erleichtern, wie beispielsweise die Kontrolle des Abgabezeitpunktes von Studierendenarbeiten oder eine geforderte Anzahl von Beiträgen in einem Forum. Die rein quantitative Auswertung der Anzahl von Beiträgen in Lernforen oder Lernchats ist mit Hilfe der Administrationswerkzeuge einfach möglich, für die Leistungsbeurteilung aber nicht unbedingt sinnvoll. Die Erfahrungen mit diesen Werkzeugen haben gezeigt, dass die Studierenden sehr rasch ihr Verhalten an rein quantitative Auswertungsmethoden anpassen und vor allem dann Quantität produzieren.

Neuere Entwicklungen zum Schutz der Privatsphäre in Informations- und Kommunikationssystemen setzen vermehrt auf den Systemdatenschutz. Die technischen Systeme sollen so gestaltet werden, dass der Personenbezug der Daten auf das notwendigste beschränkt wird. Damit wird der Interessensausgleich zwischen Informationszugang und Datenschutz bei der Technikgestaltung zu einer ständigen Herausforderung. 


\section{Literatur}

Aehnelt, M., 2003, Personalisierung als Schlüssel zum Erfolg, in: Hambach, S., Urban, B. (Hrsg.), Multimedia \& Bildung: Beiträge zu den 4. IuK-Tagen Mecklenburg-Vorpommern, Stuttgart: IRB Verlag, 129-140.

Aimeur, E., Onana, F., Seleman, A., 2006, SPRITS: Secure Pedagogical Resources in Intelligent Tutoring Systems, in: Ikeda, M, Ashley, K., Chan, T.-W. (Eds): IST 2006, Berlin, Heidelberg: Springer Verlag, 237-247.

Appelt, W., 2004, Plattformen. In: Haake, J., Schwabe, G., Wessner, M. (Hrsg.): CSCL - Kompendium. Lehr- und Handbuch zum computerunterstützten kooperativen Lernen. München: Oldenbourg Wissenschaftsverlag, 137-153.

Ariss, S. S., 2002, Computer monitoring: benefits and pitfalls facing management, Information \& Management 39, 553-558.

Baumgartner, P., Häfele, H., 2004, Lernplattformen für das Corporate e-Learning, in: Hugl, U., Laske, S., Virtuelle Personalentwicklung. Status und Trends IuKT-gestützten Lernens, Wiesbaden: Gabler Edition Wissenschaft, 95-117.

Baumgartner, P., Häfele, H., Maier-Häfele, K., 2002, E-Learning Praxishandbuch: Auswahl von Lernplattformen: Marktübersicht - Funktionen - Fachbegriffe. Innsbruck: Studienverlag.

Berthold, O., Freytag, J. C., 2004, Privacy, Datenbank-Spektrum 11, 41-44.

Bundesverfassungsgericht, 1983, Entscheidungssammlung, BVerfGE 65, 1 - Volkszählung, Abschn. II 1a.

Fischer, M., Grollmann, P., Roy, B., Steffen, N., 2003, E-Learning in der Berufsbildungspraxis: Stand, Probleme, Perspektiven, Forschungsbericht 06/2003, Bremen: Institut für Technik und Bildung.

Hampel, T., Keil-Slawik, R., Selke, H., 2004, Semantische Räume - Von der Navigation zur kooperativen Wissensstrukturierung, in: Keil-Slawik, R., Selke, H., Szwillus, G. (Hrsg.): Mensch \& Computer 2004: Allgegenwärtige Interaktion, München: Oldenbourg Verlag, 221-230.

Hansen, H.,R., Neumann, G., 2005, Wirtschaftsinformatik 1, Grundlagen und Anwendungen, 9. Auflage, Stuttgart: Lucius \& Lucius Verlagsgesellschaft.

Hippner, H., Rentzmann, R., 2006, TextMining, Informatik Spektrum 29(4), 287-290.

Hoffmann, M., 2004, Awareness und Adoption kooperativer Wissensmedien im Kontext informeller Zusammenarbeit, Dissertation, Universität Dortmund.

Identity- und Accessmanagement, freie Wissensdatenbank 2006, <http://www.iam-wiki.org>.

Jerman-Blažič, B., Klobučar, T., 2005, Privacy Provision in E-learning Standardized Systems: Status and Improvements, Computer Standards \& Interfaces 27, 561-578.

Karat, J., Carat, C. M., Brody, C., Feng, J., 2005, Privacy in information technology: designing to enable privacy policy management in organizations. International Journal of Human-Computer Studies 63,153-174.

Kerksen, S., 2003, Kompendium der Informationstechnik, Bonn: Galileo Press. 
Kobsa, A., 2004, Adaptive Verfahren - Benutzermodellierung, in: Kuhlen, R., Seeger, T., Strauch, D., (Hrsg.).: Grundlagen der praktischen Information und Dokumentation, 5. Auflage. München: K. G. Saur, 299-302.

Kuhlen, R., 1999, Die Konsequenzen der Informationsassistenten. Was bedeutet informationelle Autonomie oder wie kann Vertrauen auf elektronischen Märkten gesichert werden? Frankfurt: Suhrkamp-Verlag.

Langheinrich, M., 2005, Die Privatsphäre im Uniquitous Computing - Datenschutzaspekte der RFID-Technologie, in: Fleisch, E., Matern, M. (Hrsg.), 2005, Das Internet der Dinge, Berlin: Springer Verlag, 329-362.

Laudon, C. L., Laudon, J. P., Schoder, D., 2006, Wirtschaftsinformatik. Eine Einführung, München: Pearson Studium.

Pankoke-Babatz, U., Prinz, W., Schäfer, L., 2004, Was gibt's Neues? Asynchrone Gewärtigkeit, in: Keil-Slawik, R., Selke, H., Szwillus, G. (Hrsg.): Mensch \& Computer 2004: Allgegenwärtige Interaktion, München: Oldenbourg Verlag, 271-280.

Peissl, W., 2003, Privacy in Österreich. Eine Bestandsaufnahme. In: Peissl, W. (Hrsg.): Privacy. Ein Grundrecht mit Ablaufdatum? Wien: Verlag der Österreichischen Akademie der Wissenschaften, 155-179.

Pils, M., 2004, Electronic Business und Sensible Informations- und Kommunikationssysteme. In: Höller, J., Pils, M., Zlabinger, R. (Hrsg.): Internet und Intranet. Herausforderung EBusiness, 3. Auflage, Berlin, Heidelberg: Springer Verlag, 327-359.

Privacy Rights Clearinghouse/UCAN, A Review of the Fair Information Principles: The Foundation of Privacy Public Policy. 13.11.2006.

URL: http://www.privacyrights.org/ar/fairinfo.htm.

Reichling, T., Becks, A., Bresser, O., Wulf, V., 2004, Kontaktanbahnung in Lernplattformen, in: Keil-Slawik, R., Selke, H., Szwillus, G. (Hrsg.): Mensch \& Computer 2004: Allgegenwärtige Interaktion, München: Oldenbourg Verlag, 179-188.

Roßnagel, A., 2005, Verantwortung für Datenschutz, in: Informatik Spektrum 1, Dezember 2005, 462-473.

Schneider, F., Schauer, H., 2004, Konzepte zur Administration. In: Haake, J., Schwabe, G., Wessner, M. (Hg.): CSCL - Kompendium. Lehr- und Handbuch zum computerunterstützten kooperativen Lernen. München: Oldenbourg Wissenschaftsverlag, 154-165.

Seufert, S., 2005, Hard- und Software für E-Learning auswählen, in: Hohenstein, A., Wilbers, K. (Hrsg.): Handbuch E-Learning. Expertenwissen aus Wissenschaft und Praxis. München: Deutscher Wirtschaftsdienst.

Zweig, D., Webster, J., 2003, Personality as a moderator of monitoring acceptance, in: Computers in Human Behavior 19 (2005), 479-493. 


\section{Bisher erschienene manu:scripte}

ITA-0I-0I Gunther Tichy, Walter Peissl (12/2001): Beeinträchtigung der Privatsphäre in der Informationsgesellschaft. <http://www.oeaw.ac.at/ita/pdf/ita_0I_0I.pdf>

ITA-0I-02 Georg Aichholzer(12/2001): Delphi Austria: An Example of Tailoring Foresight to the Needs of a Small Country. <http://www.oeaw.ac.at/ita/pdf/ita_0I_02.pdf>

ITA-0I-03 Helge Torgersen, Jürgen Hampel (12/2001): The Gate-Resonance Model: The Interface of Policy, Media and the Public in Technology Conflicts.

<http://www.oeaw.ac.at/ita/pdf/ita_0l_03.pdf>

ITA-02-0I Georg Aichholzer (01/2002): Das ExpertInnen-Delphi: Methodische Grundlagen und Anwendungsfeld ,Technology Foresight“. <http://www.oeaw.ac.at/ita/pdf/ita_02_0I.pdf>

ITA-02-02 Walter Peissl (01/2002): Surveillance and Security - A Dodgy Relationship. $<$ http://www.oeaw.ac.at/ita/pdf/ita_02_02.pdf $>$

ITA-02-03 Gunther Tichy (02/2002): Informationsgesellschaft und flexiblere Arbeitsmärkte. $<$ http://www.oeaw.ac.at/ita/pdf/ita_02_03.pdf >

ITA-02-04 Andreas Diekmann (06/2002): Diagnose von Fehlerquellen und methodische Qualität in der sozialwissenschaftlichen Forschung. <http://www.oeaw.ac.at/ita/pdf/ita_02_04.pdf>

ITA-02-05 Gunther Tichy (10/2002): Over-optimism Among Experts in Assessment and Foresight. <http://www.oeaw.ac.at/ita/pdf/ita_02_05.pdf>

ITA-02-06 Hilmar Westholm (12/2002): Mit eDemocracy zu deliberativer Politik? Zur Praxis und Anschlussfähigkeit eines neuen Mediums. <http://www.oeaw.ac.at/ita/pdf/ita_02_06.pdf>

ITA-03-0I Jörg Flecker und Sabine Kirschenhofer (01/2003): IT verleiht Flügel? Aktuelle Tendenzen der räumlichen Verlagerung von Arbeit. $<$ http://www.oeaw.ac.at/ita/pdf/ita_03_0l.pdf>

ITA-03-02 Gunther Tichy (11/2003): Die Risikogesellschaft - Ein vernachlässigtes Konzept in der europäischen Stagnationsdiskussion.

$<$ http://www.oeaw.ac.at/ita/pdf/ita_03_02.pdf>

ITA-03-03 Michael Nentwich (11/2003): Neue Kommunikationstechnologien und Wissenschaft - Veränderungspotentiale und Handlungsoptionen auf dem Weg zur Cyber-Wissenschaft. <http://www.oeaw.ac.at/ita/pdf/ita_03_03.pdf>

ITA-04-0I Gerd Schienstock (1/2004): Finnland auf dem Weg zur Wissensökonomie - Von Pfadabhängigkeit zu Pfadentwicklung. <http://www.oeaw.ac.at/ita/pdf/ita_04_0I.pdf>

ITA-04-02 Gunther Tichy (6/2004): Technikfolgen-Abschätzung: Entscheidungshilfe in einer komplexen Welt. <http://www.oeaw.ac.at/ita/pdf/ita_04_02.pdf>

ITA-04-03 Johannes M. Bauer (11/2004): Governing the Networks of the Information Society - Prospects and limits of policy in a complex technical system.

<http://www.oeaw.ac.at/ita/pdf/ita_04_03.pdf>

ITA-04-04 Ronald Leenes (12/2004): Local e-Government in the Netherlands: From Ambitious Policy Goals to Harsh Reality. <http://www.oeaw.ac.at/ita/pdf/ita_04_04.pdf>

ITA-05-0I Andreas Krisch (01/2005): Die Veröffentlichung des Privaten - Mit intelligenten Etiketten vom grundsätzlichen Schutz der Privatsphäre zum Selbstschutz-Prinzip. <http://www.oeaw.ac.at/ita/pdf/ita_05_0I.pdf> 
ITA-05-02 Petra Grabner (12/2005): Ein Subsidiaritätstest - Die Errichtung gentechnikfreier Regionen in Österreich zwischen Anspruch und Wirklichkeit.

<http://www.oeaw.ac.at/ita/pdf/ita_05_02.pdf>

ITA-05-03 Eva Buchinger (12/2005): Innovationspolitik aus systemtheoretischer Sicht - Ein zyklisches Modell der politischen Steuerung technologischer Innovation. $<$ http://www.oeaw.ac.at/ita/pdf/ita_05_03.pdf>

ITA-06-0I Michael Latzer (06/2006): Medien- und Telekommunikationspolitik: Unordnung durch Konvergenz - Ordnung durch Mediamatikpolitik. $<$ http://epub.oeaw.ac.at/ita/ita-manuscript/ita_06_0I.pdf $>$

ITA-06-02 Natascha Just, Michael Latzer, Florian Saurwein (09/2006): Communications Governance: Entscheidungshilfe für die Wahl des Regulierungsarrangements am Beispiel Spam. <http://epub.oeaw.ac.at/ita/ita-manuscript/ita_06_02.pdf >

ITA-06-03 Veronika Gaube, Helmut Haberl (10/2006): Sozial-ökologische Konzepte, Modelle und Indikatoren nachhaltiger Entwicklung: Trends im Ressourcenverbrauch in Österreich. $<$ http://epub.oeaw.ac.at/ita/ita-manuscript/ita_06_03.pdf>

ITA-06-04 Maximilian Fochler, Annina Müller (11/2006): Vom Defizit zum Dialog? Zum Verhältnis von Wissenschaft und Öffentlichkeit in der europäischen und österreichischen Forschungspolitik.

$<$ http://epub.oeaw.ac.at/ita/ita-manuscript/ita_06_04.pdf>

ITA-06-05 Holger Floeting (11/2006): Sicherheitstechnologien und neue urbane Sicherheitsregimes. $<$ http://epub.oeaw.ac.at/ita/ita-manuscript/ita_06_05.pdf>

ITA-06-06 Armin Spök (12/2006): From Farming to "Pharming" - Risks and Policy Challenges of Third Generation GM Crops. <http://epub.oeaw.ac.at/ita/ita-manuscript/ita_06_06.pdf>

ITA-07-0I Volker Stelzer, Christine Rösch, Konrad Raab (3/2007): Ein integratives Konzept zur Messung von Nachhaltigkeit - das Beispiel Energiegewinnung aus Grünland. $<$ http://epub.oeaw.ac.at/ita/ita-manuscript/ita_07_0I.pdf $>$

ITA-07-02 Elisabeth Katzlinger (3/2007): Big Brother beim Lernen: Privatsphäre und Datenschutz in Lernplattformen.

$<$ http://epub.oeaw.ac.at/ita/ita-manuscript/ita_07_02.pdf $>$ 